\title{
CHASING HIGHLY OBSCURED QSOs IN THE COSMOS FIELD
}

\author{
F. Fiore ${ }^{1}$, S. Puccetti ${ }^{2}$, M. Brusa ${ }^{3}$, M. Salvato ${ }^{4}$, G. Zamorani ${ }^{5}$, T. Alderoft ${ }^{6}$, H. Aussel ${ }^{7}$, H. Brunner 3 , P. Capak ${ }^{4}$, \\ N. Cappelluti ${ }^{3}$, F. Civano ${ }^{6}$, A. Comastri ${ }^{5}$, M. Elvis ${ }^{6}$, C. Feruglio $^{7}$, A. Finoguenov ${ }^{3}$, A. Fruscione ${ }^{6}$, R. Gillit ${ }^{5}$,

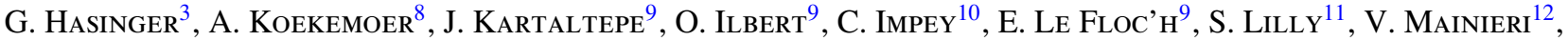 \\ A. Martinez-Sansigre ${ }^{13}$, H. J. McCracken ${ }^{14}$, N. Menci ${ }^{1}$, A. Merloni ${ }^{3}$, T. Miyaji ${ }^{15}$, D. B. Sanders ${ }^{9}$, M. Sargent ${ }^{13}$, \\ E. Schinnerer ${ }^{13}$, N. Scovillee ${ }^{4}$, J. Silverman ${ }^{11}$, V. Smolcic ${ }^{14}$, A. StefFen ${ }^{4}$, P. Santini ${ }^{1}$, Y. Taniguchi ${ }^{16}$, D. Thompson ${ }^{4}, 17$, \\ J. R. Trump ${ }^{10}$, C. Vignali ${ }^{18}$, M. URrY ${ }^{19}$, AND L. YAN ${ }^{4}$ \\ ${ }^{1}$ INAF-Osservatorio Astronomico di Roma, via Frascati 33, Monteporzio (Rm), I00040, Italy; fiore@oa-roma.inaf.it \\ ${ }^{2}$ ASI Science Data Center, via Galileo Galilei, 00044 Frascati, Italy \\ ${ }^{3}$ Max-Planck-Institut für Extraterrestrische Physik, Giessenbachstrasse 1, D-85748 Garching, Germany \\ ${ }^{4}$ California Institute of Technology, MC 105-24, 1200 East California Boulevard, Pasadena, CA 91125, USA \\ ${ }^{5}$ INAF-Osservatorio Astronomico di Bologna, via Ranzani 1, I-40127 Bologna, Italy \\ ${ }^{6}$ Harvard-Smithsonian Center for Astrophysics, 60 Garden Street, Cambridge, MA 02138, USA \\ ${ }^{7}$ CEA/DSM-CNRS, Universite' Paris Diderot, DAPNIA/SAp, Orme des Merisiers, 91191, Gif-sur-Yvette, France \\ ${ }^{8}$ Space Telescope Science Institute, 3700 SanMartin Drive, Baltimore, MD 21218, USA \\ ${ }^{9}$ Institute for Astronomy, University of Hawaii, 2680 Woodlawn Drive, Honolulu, HI, 96822, USA \\ ${ }^{10}$ Steward Observatory, University of Arizona, Tucson, AZ 85721, USA \\ ${ }^{11}$ Department of Physics, Eidgenossiche Technische Hochschule (ETH), CH-8093 Zurich, Switzerland \\ ${ }^{12}$ European Southern Observatory, Karl-Schwarzschild-str. 2, 85748 Garching bei München, Germany \\ ${ }^{13}$ Max-Planck-Institut für Astronomie, Königstuhl 17, D-69117 Heidelberg, Germany \\ ${ }^{14}$ Institut d'Astrophysique de Paris, UMR7095 CNRS, Université Pierre \& Marie Curie, 98 bis Boulevard Arago, 75014 Paris, France \\ 15 1IA-UNAM-Ensenada, Mexico \\ ${ }^{16}$ Department of Physics, Ehime University, 2-5 Bunkyo-cho Matsuyama 790-8577, Japan \\ ${ }^{17}$ Large Binocular Telescope Observatory, University of Arizona, 933 N. Cherry Ave., Tucson, AZ 85721-0065, USA \\ ${ }^{18}$ Universita' di Bologna, via Ranzani 1, Bologna, Italy \\ ${ }^{19}$ Yale Center for Astronomy and Astrophysics, Yale University, P.O. Box 208121, New Haven, CT 06520-8121, USA \\ Received 2008 May 25; accepted 2008 October 20; published 2009 March 2
}

\begin{abstract}
A large population of heavily obscured, Compton-thick active galactic nuclei (AGNs) is predicted by AGN synthesis models for the cosmic X-ray background and by the "relic" supermassive black hole mass function measured from local bulges. However, even the deepest X-ray surveys are inefficient to search for these elusive AGNs. Alternative selection criteria, combining mid-infrared with near-infrared, and optical photometry, have instead been successful in pinpointing a large population of Compton-thick AGNs. We take advantage of the deep Chandra and Spitzer coverage of a large area (more than 10 times the area covered by the Chandra deep fields, CDFs) in the Cosmic Evolution Survey (COSMOS) field to extend the search of highly obscured, Compton-thick active nuclei to higher luminosity. These sources have low surface density, and therefore large samples can be provided only through large area surveys, like the COSMOS survey. We analyze the X-ray properties of COSMOS MIPS sources with $24 \mu \mathrm{m}$ fluxes higher than $550 \mu \mathrm{Jy}$. For the MIPS sources not directly detected in the Chandra images, we produce stacked images in soft and hard X-rays bands. To estimate the fraction of Compton-thick AGN in the MIPS source population, we compare the observed stacked count rates and hardness ratios to those predicted by detailed Monte Carlo simulations, including both obscured AGN and star-forming galaxies. The volume density of Compton-thick QSOs $\left(\log \mathrm{L}(2-10 \mathrm{keV})=44-45 \mathrm{erg} \mathrm{s}^{-1}\right.$, or $\log \lambda L_{\lambda}(5.8 \mu \mathrm{m})=44.79-46.18 \mathrm{erg} \mathrm{s}^{-1}$ for a typical infrared to X-ray luminosity ratio) evaluated in this way is $(4.8 \pm 1.1) \times 10^{-6} \mathrm{Mpc}^{-3}$ in the redshift bin $1.2-2.2$. This density is $\sim 44 \%$ of all X-ray-selected QSOs in the same redshift and luminosity bin, and it is consistent with the expectation of the most up-to-date AGN synthesis models for the cosmic X-ray background (Gilli et al. 2007). The density of lower luminosity Compton-thick AGNs $(\log \mathrm{L}(2-10 \mathrm{keV})=43.5-44)$ at $z=0.7-1.2$ is $(3.7 \pm 1.1) \times 10^{-5} \mathrm{Mpc}^{-3}$, corresponding to $\sim 67 \%$ of X-ray-selected AGNs. The comparison between the fraction of infrared-selected, Compton-thick AGNs to the X-ray selected, unobscured, and moderately obscured AGNs at high and low luminosity suggests that Compton-thick AGNs follow a luminosity dependence similar to that discovered for Compton-thin AGNs, becoming relatively rarer at high luminosities. We estimate that the fraction of AGNs (unobscured, moderately obscured, and Compton thick) to the total MIPS source population is $49 \pm 10 \%$, a value significantly higher than that previously estimated at similar $24 \mu \mathrm{m}$ fluxes. We discuss how our findings can constrain AGN feedback models.
\end{abstract}

Key words: galaxies: active - galaxies: high-redshift - X-rays: diffuse background

Online-only material: color figures

\section{INTRODUCTION}

Understanding how galaxies formed and how they became the complex systems we observe in the local Universe is a major theoretical and observational effort, mainly pursued using large and deep multiwavelength surveys. The ubiquitous observation of "relic" supermassive black holes (SMBHs) in the center of nearby bulge-dominated galaxies, and the discovery of tight 
correlations between their masses and bulge properties suggest strong links and feedbacks between SMBHs, nuclear activity, and galaxy evolution (Gebhardt et al. 2000; Ferrarese \& Merritt 2000 and references therein). The peak of both nuclear (active galactic nucleus, AGN) and star-formation activities is at $z \gtrsim 1$ (Boyle et al. 1988; Madau et al. 1996; Hopkins 2006; Brandt \& Hasinger 2005 and references therein), possibly due to the fact that more gas is available at high- $z$ for both AGN fueling and star formation. In recent years, much evidence has been accumulated showing similar mass-dependent evolutions for galaxies and AGNs (i.e., black holes). We have robust evidence that massive galaxies are characterized by a star-formation history that peaks at $z \gtrsim 2$ (Renzini 2006), while lower mass galaxies are typically younger systems (Cowie et al. 1996). Similarly, the density of the high luminosity AGNs (QSOs hereinafter, see Table 1 for a quantitative definition) peaks at $z \gtrsim 2$ and declines strongly afterward, while lower luminosity AGNs follow a much smoother behavior, peaking at lower redshifts, $z=1-1.5$ (Ueda et al. 2003; Cowie et al. 2003; Fiore et al. 2003; Hasinger et al. 2005; La Franca et al. 2005). These trends in the evolution of galaxies and AGNs have been dubbed "downsizing" (e.g., Cowie et al. 1996; Franceschini et al. 1999), meaning that large structures tend to have formed earlier and have grown faster than smaller structures. The coevolution of galaxies and AGNs and their downsizing depends on feedback between nuclear and galactic activities (Silk \& Rees 1998; Fabian 1999; Granato et al. 2001, 2004; Menci et al. 2006; Bower et al. 2006; Daddi et al. 2007). QSOs, presumably hosted in high mass progenitors, must somehow be more efficient at inhibiting star formation in their host galaxies, by heating the interstellar matter through winds, shocks, and high energy radiation (Silk \& Rees 1998; Fabian 1999; Granato et al. 2001, 2004; Di Matteo et al. 2005; Menci et al. 2006, 2008; Bower et al. 2006; Li et al. 2007). In this scenario, a short, powerful AGN phase is believed to precede the phase when a galaxy is found in a passive status with red optical and UV colors, most of the star formation having been inhibited by the AGN activity. Conversely, feedback from less powerful AGNs, presumably hosted in low mass progenitors, is more effective in self-regulating accretion and star formation, and cold gas is left available for both processes for a much longer time. The same cold gas can intercept the line of sight to the nucleus, and therefore a natural expectation of this scenario is that the fraction of obscured AGNs to the total AGN population is large at low AGN luminosities and decreases at high luminosities, as it is indeed observed (Lawrence \& Elvis 1982; Ueda et al. 2003; Steffen et al. 2003; La Franca et al. 2005; Treister \& Urry 2005, 2006; Maiolino et al. 2007; Hasinger 2008; Trump et al. 2009; Della Ceca et al. 2008). Powerful AGNs clean their sight lines more quickly than low luminosity AGNs, and therefore the fraction of active objects caught in an obscured phase decreases with the luminosity. Under this hypothesis, the fraction of obscured AGNs can be viewed as a measure of the timescale over which the nuclear feedback is at work (Menci et al. 2008). In this respect, the trend of the fraction of obscured AGNs with the luminosity can be regarded as a manifestation of the AGN downsizing. Obscured AGNs are thus laboratories where feedback in action is investigated.

We call objects with their SMBH in an active status "AGNs". This does not imply that the AGNs must dominate the bolometric luminosity, but simply that it is possible to recognize its emission in at least one of the bands of the electromagnetic spectrum. We call those AGNs in which the optical and soft X-ray nuclear light is not blocked by gas and dust along the line
Table 1

AGN Definitions Used in This Paper

\begin{tabular}{lcc}
\hline \hline AGN Type & $\mathrm{A}_{V}$ & $\begin{array}{c}\log \mathrm{N}_{H} \\
\mathrm{~cm}^{-2}\end{array}$ \\
\hline Unobscured & $<5^{\mathrm{a}}$ & $\begin{array}{c}\lesssim 22 \\
\text { Moderately obscured (Compton thin) }\end{array}$ \\
Highly obscured (Compton thick) & $>5$ & $22-24$ \\
\hline AGN type & $\log \mathrm{L}(2-10 \mathrm{keV})$ & $>24$ \\
& $\mathrm{erg} \mathrm{cm}^{-2} \mathrm{~s}^{-1}$ & $\begin{array}{c}\log \mathrm{N}_{H} \\
\mathrm{~cm}^{-2}\end{array}$ \\
\hline Unobscured QSO & $>44$ & $\lesssim 22$ \\
Compton-thin QSO & $>44$ & $22-24$ \\
Compton-thick QSO & $>44$ & $>24$ \\
\hline
\end{tabular}

Notes.

${ }^{a}$ From Simpson et al. (1999).

b Allowing for the fact that obscured AGNs and QSOs can have gas-to-dust ratios much smaller than the galactic value (see, e.g., Maiolino et al. 2001; Martinez-Sansigre et al. 2006).

of sight "unobscured". AGNs in which the nuclear light is blocked or reduced by dust and gas along the line of sight are called "obscured." We further distinguish between moderately obscured AGNs (or Compton-thin) and highly obscured AGNs (or Compton thick, CT) (see Table 1 for quantitative definitions). While unobscured and moderately obscured AGNs can be efficiently selected in current X-ray surveys, even the deepest Chandra and XMM-Newton surveys detected only a handful of CT AGNs directly (e.g., Tozzi et al. 2006; Comastri 2004). However, the SMBH mass function obtained by integrating these X-ray luminosity functions falls short by a factor $\sim 1.5-2$ (depending on the assumed efficiency in the conversion of gravitational energy into radiation) of the SMBH mass function, evaluated using the $M_{\mathrm{BH}}-\sigma_{V} / M_{\mathrm{BH}}-\mathrm{M}_{B}$ relationships and the local bulge's luminosity function (the "relic" SMBH mass function, Marconi et al. 2004, but also see Merloni \& Heinz 2008). AGN synthesis models for the cosmic X-ray background (CXB, Treister et al. 2004; Treister \& Urry 2005; Gilli et al. 2007) predict a large enough volume density of CT AGNs to reconcile the "active" and "relic" SMBH mass functions.

Obscured AGNs, including CT ones, can be recovered, thanks to the reprocessing of the AGN UV emission in the infrared, by selecting sources with mid-infrared (and/or radio) AGN luminosities but faint near infrared and optical emission. Houck et al. (2005), Weedman et al. (2006a, 2006b), Yan et al. (2007), and Polletta et al. (2008) obtained Spitzer IRS spectra of large samples of relatively bright $24 \mu \mathrm{m}$ sources $(F(24 \mu \mathrm{m})>0.7 \mathrm{mJy})$ with faint optical counterparts, finding that the majority are AGN dominated. The small UV rest frame luminosity implies significant obscuration in these objects. Indeed, Polletta et al. (2006) used X-ray data to infer that some of these infrared bright QSOs are Compton thick. Martinez-Sansigre et al. (2005, 2007, 2008) obtained optical and Spitzer IRS spectra of sources with $F(24 \mu \mathrm{m})>0.3 \mathrm{mJy}$, and faint optical and near-infrared counterparts, finding that most are highly obscured type 2 QSOs. Brand et al. (2007) obtained infrared spectroscopy of 10 sources with $F(24 \mu \mathrm{m})>0.8 \mathrm{mJy}$ and faint optical counterparts, finding that six exhibit broad $\mathrm{H} \alpha$ lines. Since both the narrow line region and the UV continuum are strongly extinted, they suggest that the obscuration is due to dust on large scales within the host galaxies. Dey et al. (2008) obtained optical spectroscopy of a rather large sample of objects with extreme $F(24 \mu \mathrm{m}) / F(R)$ flux ratios and $F(24 \mu \mathrm{m})>0.3 \mathrm{mJy}$. They found a redshift distribution centered at $z \sim 2$, implying large luminosities, and concluded that both star formation and nuclear activity are 
Table 2

Main Datasets Used in This Paper

\begin{tabular}{lcccccc}
\hline \hline Dataset & Area & Total Exposure & Typical Exposure & FWHM & Detected Sources & Flux Limit \\
\hline C-COSMOS & $0.9 \mathrm{deg}^{2}$ & $1.8 \mathrm{Ms}$ & $90-185 \mathrm{ks}^{\mathrm{a}}$ & $2^{\prime \prime} \mathrm{b}$ & 1760 & $2 \times 10^{-16 \mathrm{c}}$ \\
S-COSMOS (MIPS) & $0.9 \mathrm{deg}^{2}$ & $58.2 \mathrm{hr}$ & $80 \mathrm{sec}$ & $5^{\prime \prime \mathrm{d}}$ & 919 & $550 \mu \mathrm{Jy}$ \\
\hline
\end{tabular}

Notes.

$\mathrm{a} \sim$ half of the total area is covered with an effective exposure of $\sim 185 \mathrm{ks}$, the remaining half has an effective exposure of $\sim 90 \mathrm{ks}$.

$\mathrm{b}$ average for four overlapping fields.

${ }^{c} \operatorname{ergcm}^{-2} \mathrm{~s}^{-1} 0.5-2 \mathrm{keV}$.

d PSF core.

probably contributing to these luminosities. Finally, Daddi et al. (2007) and Fiore et al. (2008, F08 hereafter) and Georgantopoulos et al. (2008) suggested that the majority of the so-called "IR excess" sources in the Chandra deep fields (CDFs), with an extreme $F(24 \mu \mathrm{m}) / F(R)$ flux ratios and $F(24 \mu \mathrm{m})$ as low as $40 \mu \mathrm{Jy}$, are highly obscured, possibly CT AGNs at $z=1-3$. Although Donley et al. (2008) and Pope et al. (2008) disagree with the latter two studies on the AGN fraction at faint $24 \mu \mathrm{m}$ fluxes, it is clear that selecting bright $24 \mu \mathrm{m}$ sources with extreme $F(24 \mu \mathrm{m}) / F(R)$ flux ratios may represent a promising method to complement X-ray surveys in obtaining sizable samples of CT AGNs, and so completing the census of accreting SMBHs at these redshifts.

Here we apply and extend this approach to the Cosmic Evolution Survey (COSMOS) field to estimate the total (unobscured, moderately obscured, and CT) AGN fraction to the full MIPS $24 \mu \mathrm{m}$ galaxy population. We take advantage of its deep and uniform coverage at infrared, optical, and X-ray wavelengths to select and validate samples of CT AGNs at $z=0.7-2$. The COSMOS sample contains sources that have IR/optical properties similar to those in the CDFs, but are $\sim 10$ times brighter, and are therefore much more luminous than the CDFs AGNs at the same redshift. Our goal is to select a sizable sample of high luminosity CT QSOs to accurately measure their volume density and to understand whether their obscuration properties are similar to those of lower luminosity AGNs. This will allow us to understand whether the correlation between the fraction of obscured AGNs and luminosity holds for CT QSOs, and to extend this study up to $z \sim 2$. Such luminous sources are rare, and only taking advantage of the large area covered by COSMOS they can be found in significant number to make statistical studies. The total area covered by COSMOS is $2 \mathrm{deg}^{2}$, but in this work we limit the analysis to the area covered by deep Chandra observations ( $\sim 0.9 \mathrm{deg}^{2}, \gtrsim 10$ times the area in CDFs).

This paper is organized as follows. Section 2 presents the datasets used in this work and the selection of the CT QSO sample from the Spitzer MIPS $24 \mu \mathrm{m}$ COSMOS sample; Section 3 discusses the X-ray properties of the MIPS-selected sources. Section 4 presents the result of fitting the broad band spectral energy distributions (SEDs) of the MIPS-selected sources with galaxy and AGN templates. Section 5 presents our evaluation of the infrared-selected CT QSO volume density. Finally, Section 6 gives our conclusions. A $H_{0}=70 \mathrm{~km} \mathrm{~s}^{-1} \mathrm{Mpc}^{-1}, \Omega_{M}=0.3$, $\Omega_{\Lambda}=0.7$ cosmology is adopted throughout.

\section{DATASETS AND SAMPLE SELECTION}

The COSMOS field (Scoville et al. 2007) is so far a unique area for its deep and wide, comprehensive multiwavelength coverage, from the optical band with Hubble and ground based $8 \mathrm{~m}$ class telescopes, to infrared with Spitzer, to X-rays with
XMM-Newton and Chandra, to the radio with the Very Large Array (VLA). The COSMOS field samples a volume at high redshift $(z \lesssim 2)$ that is $\sim 15 \%$ of that sampled by SDSS at $z \lesssim 0.15$.

For this work, we use the Spitzer MIPS $24 \mu \mathrm{m}$ COSMOS catalog derived from cycle 2 shallow observations (Sanders et al. 2007). Here, we do not use the cycle 2 deep MIPS test area because it covers only a small fraction of the Chandra-COSMOS area. The cycle 2 catalog has been cleaned of spurious sources (mostly asteroids) by comparison with the much deeper MIPS mosaic obtained in cycle 3 (H. Aussel et al. 2009, in preparation), a reliable catalog from this mosaic is in preparation and will be used in a follow-up publication). We consider only MIPS $24 \mu \mathrm{m}$ sources with a signal-to-noise ratio $>4$, implying a $24 \mu \mathrm{m}$ flux limit of $\sim 550 \mu \mathrm{Jy}$. We limit the analysis to the area covered by Chandra observations $\left(\sim 0.9 \mathrm{deg}^{2}\right.$, centered on R.A. $=1000$ 20, decl. = +02 09 28; Elvis et al. 2009). The sample includes 919 sources, and we refer to this sample in the following as the COSMOS bright MIPS sample (see Table 2 for further detail).

The Spitzer MIPS catalog has been cross correlated with the IRAC catalog ${ }^{20}$ (Sanders et al. 2007), the optical multiband catalog (Capak et al. 2007), and the K-band catalog (H. McCracken et al. 2009, in preparation). IRAC, K band, and optical counterparts of the MIPS sources have been carefully identified with a 2-step approach: first, each MIPS source has been associated with the most likely IRAC counterpart within 2 arcsec from the MIPS centroid, then IRAC positions have been correlated with the optical and K-band catalogs with a matching radius of 0.5 arcsec. A total of 859 sources of the COSMOS bright MIPS catalog have counterparts in all IRAC, optical, and $\mathrm{K}$ bands, and 53 sources have counterparts in only one or two catalogs. Finally, four sources have optical counterparts too close to a bright source (and therefore no reliable photometry is available), while for the remaining three sources no optical counterpart was assigned (they are either residual asteroids or too faint to be detected).

The X-ray properties of the MIPS-selected sources have been studied using the Chandra data. Chandra observed the COSMOS field for $1.8 \mathrm{Ms}$. The survey uses a series of 36 heavily overlapped ACIS-I $50 \mathrm{ks}$ pointings to give an unprecedented uniform effective exposure of $185 \mathrm{ks}$ over a large area. Particular care was taken in performing accurate astrometric corrections and in the reduction of the internal background (see Elvis et al. 2009 for details). The residual background is very stable over the full field at $\lesssim 2$ counts/200 ks over an area of 2 arcsec radius, comparable with the Chandra beam size (see Table 2 for further details). Uniform coverage and low background make the Chandra COSMOS (C-COSMOS) dataset ideal for stacking analyses.

\footnotetext{
${ }^{20} \mathrm{http}$ ///irsa.ipac.caltech.edu/cgi-bin/Gator/nph-scansubmit=Select\& projshort=COSMOS
} 
Table 3

MIPS Sources With a Direct Chandra Detection

\begin{tabular}{lcccccccc}
\hline \hline Cell & $F(24 \mu \mathrm{m}) / F(R)$ & $\mathrm{R}-\mathrm{K}$ & $\begin{array}{c}\text { \# of MIPS } \\
\text { Sources }\end{array}$ & \# of X-ray det. & $\langle z\rangle$ & $\begin{array}{c}\langle\log \mathrm{L}(5.8 \mu \mathrm{m})\rangle \\
\mathrm{erg} \mathrm{s}^{-1}\end{array}$ & $\begin{array}{c}\langle\log \mathrm{L}(2-10 \mathrm{keV})\rangle \\
\mathrm{erg} \mathrm{s}^{-1}\end{array}$ \\
\hline $\mathrm{A}$ & $>1000$ & $>4.5$ & 73 & $31(40 \%)$ & $1.55 \pm 0.53$ & $45.20 \pm 0.44$ & $43.51 \pm 0.40$ & $0.50 \pm 0.34$ \\
$\mathrm{~B}$ & $300-1000$ & $>4.5$ & 98 & $34(35 \%)$ & $0.96 \pm 0.13$ & $44.58 \pm 0.27$ & $43.15 \pm 0.41$ & $0.44 \pm 0.31$ \\
$\mathrm{C}$ & $100-300$ & $>4.5$ & 105 & $24(23 \%)$ & $0.73 \pm 0.12$ & $44.12 \pm 0.19$ & $42.69 \pm 0.57$ & $0.58 \pm 0.22$ \\
$\mathrm{D}$ & $10-100$ & $>4.5$ & 65 & $14(22 \%)$ & $0.50 \pm 0.25$ & $43.80 \pm 0.20$ & $42.01 \pm 0.65$ & $0.48 \pm 0.28$ \\
\hline $\mathrm{E}$ & $100-1000$ & $3-4.5$ & 72 & $26(36 \%)$ & $0.90 \pm 0.37$ & $44.61 \pm 0.46$ & $43.17 \pm 0.78$ & $0.29 \pm 0.31$ \\
$\mathrm{~F}$ & $30-100$ & $3-4.5$ & 187 & $61(33 \%)$ & $0.48 \pm 0.21$ & $43.94 \pm 0.35$ & $42.60 \pm 0.87$ & $0.00 \pm 0.22$ \\
$\mathrm{G}$ & $10-30$ & $3-4.5$ & 144 & $19(13 \%)$ & $0.17 \pm 0.07$ & $43.29 \pm 0.26$ & $40.85 \pm 0.85$ & $0.28 \pm 0.41$ \\
$\mathrm{H}$ & $1-10$ & $3-4.5$ & 14 & $4(29 \%)$ & $0.13 \pm 0.18$ & $43.16 \pm 0.68$ & $41.00 \pm 0.74$ & $0.35 \pm 0.22$ \\
\hline $\mathrm{J}$ & $10-100$ & $1-3$ & 52 & $41(79 \%)$ & $1.48 \pm 0.33$ & $45.30 \pm 0.32$ & $44.15 \pm 0.34$ & $-0.06 \pm 0.12$ \\
\hline
\end{tabular}

Note. ${ }^{\mathrm{a}} \mathrm{H}=1.5-6 \mathrm{keV}, \mathrm{S}=0.3-1.5 \mathrm{keV}$.

Table 4

MIPS Sources Without a Direct Chandra Detection

\begin{tabular}{|c|c|c|c|c|c|c|c|c|c|}
\hline Cell & $F(24 \mu \mathrm{m}) / F(R)$ & $\mathrm{R}-\mathrm{K}$ & $\begin{array}{c}\text { \# of MIPS } \\
\text { Sources }\end{array}$ & $\langle z\rangle$ & $\begin{array}{c}\langle\log \mathrm{L}(5.8 \mu \mathrm{m})\rangle \\
\mathrm{erg} \mathrm{s}^{-1}\end{array}$ & $\begin{array}{c}\text { Counts } \\
1.5-6 \mathrm{keV}\end{array}$ & $\begin{array}{c}\text { Counts } \\
0.3-1.5 \mathrm{keV}\end{array}$ & $\langle(H-S) /(H+S)\rangle^{\mathrm{a}}$ & $\begin{array}{l}\text { CT AGN } \\
\text { Fraction } \\
\end{array}$ \\
\hline A & $>1000$ & $>4.5$ & 42 & $1.90 \pm 0.40$ & 45.240 .24 & $58.9 \pm 8.5$ & $18.0 \pm 4.7$ & $0.53 \pm 0.14$ & $\overline{0.94_{-0.08}^{+0.06}}$ \\
\hline $\mathrm{C}$ & $100-300$ & $>4.5$ & 81 & $0.79 \pm 0.14$ & 44.010 .17 & $42.2 \pm 7.2$ & $36.9 \pm 6.7$ & $0.07 \pm 0.12$ & $0.51_{-0.18}^{+0.17}$ \\
\hline $\mathrm{D}$ & $10-100$ & $>4.5$ & 51 & $0.37 \pm 0.12$ & 43.760 .24 & $31.7 \pm 6.2$ & $31.1 \pm 6.2$ & $0.01 \pm 0.14$ & $0.42_{-0.23}^{+0.16}$ \\
\hline $\mathrm{E}$ & $100-1000$ & $3-4.5$ & 46 & $0.70 \pm 0.22$ & 44.060 .43 & $27.7 \pm 5.8$ & $36.1 \pm 6.6$ & $-0.13 \pm 0.14$ & $0.26_{-0.26}^{+0.29}$ \\
\hline G & $10-30$ & $3-4.5$ & 125 & $0.23 \pm 0.06$ & 43.300 .21 & $54.8 \pm 8.2$ & $108.9 \pm 11.5$ & $-0.33 \pm 0.09$ & $<0.40$ \\
\hline $\mathrm{H}$ & $1-10$ & $3-4.5$ & 10 & $0.11 \pm 0.04$ & 42.570 .29 & $1.3 \pm 1.3$ & $10.4 \pm 3.6$ & $-0.78_{-0.22}^{+0.41}$ & $<0.20$ \\
\hline $\mathbf{J}$ & $10-100$ & $1-3$ & 11 & $0.34 \pm 0.12$ & 43.520 .35 & $0.8 \pm 1.0$ & $17.7 \pm 4.6$ & $-0.92_{-0.08}^{+0.35}$ & $<0.05$ \\
\hline
\end{tabular}

Note. ${ }^{\mathrm{a}} \mathrm{H}=1.5-6 \mathrm{keV} ; \mathrm{S}=0.3-1.5 \mathrm{keV}$.

\subsection{Redshifts}

For $96 \%$ of the COSMOS bright MIPS sample, either spectroscopic or robust photometric redshifts have been obtained. Accurate spectroscopic redshifts are present for 394 MIPS sources, $43 \%$ of the sample (Lilly et al. 2007; Trump et al. 2007, 2008).

Regarding photometric redshifts, we used both the computation of Ilbert et al. (2008) and of Salvato et al. (2008), which made use of about 30 photometric data points (including 12 intermediate filters observed with SUBARU). The first concentrates on objects with $I(A B)<26.5$, with SEDs dominated by the integrated stellar population at $\lambda<5.5 \mu \mathrm{m}$. The achieved accuracy for the MIPS-selected sample is $\sigma(\Delta z /(1+z))=0.01$ for $I<24$. The accuracy degrades at $I>24(\sigma(\Delta z /(1+z))$ $=0.05)$. The second photometric redshift catalog deals with the XMM-COSMOS (Hasinger et al. 2007) sources that are dominated by AGN emission. For better results (1) correction for variability, (2) luminosity priors for point-like sources, and (3) a new set of SED templates have been adopted. Thus, for the first time, an accuracy comparable to those of photometric redshift for nonactive galaxies has been achieved $(\sigma(\Delta z /(1+z))<0.02$ and less that $5 \%$ outliers $)$. This second photometric dataset, specific for X-ray detected sources, was used when the MIPS-selected source was associated with an XMM-Newton detection. The median redshift of the COSMOS bright MIPS sample is 0.64 with interquartile 0.36 . Total 230 sources have $z>1$ and $56 z>2$. Tables 3 and 4 also give the median redshift and its interquartile of MIPS samples selected at intervals of $F(24 \mu \mathrm{m}) / F(R)$ and $\mathrm{R}-\mathrm{K}$ color (see next section).

\subsection{Optical, Near-Infrared and Mid-Infrared Color Selection}

F08 proposed a criterion, based on high mid-infrared to optical flux ratios and red optical colors, to efficiently select candidate CT AGNs, and applied this method to the CDFS area. Briefly, they selected a sample of candidate CT AGNs by using the combination of $F(24 \mu \mathrm{m}) / F(R)>1000$ and $\mathrm{R}-\mathrm{K}>4.5$ colors. They demonstrated that the selected sample was made mainly of CT AGNs through a stacking analysis of the Chandra X-ray data. The CDFS infrared-selected CT AGNs have $z \sim 1-3$, and $\lambda L_{\lambda}(5.8 \mu \mathrm{m}) \approx 10^{44-45} \mathrm{erg} \mathrm{s}^{-1}$, corresponding to intrinsic X-ray $(2-10 \mathrm{keV})$ luminosities $\approx$ $10^{43-44} \mathrm{erg} \mathrm{s}^{-1}$. F08 limited their analysis to MIPS sources with $F(24 \mu \mathrm{m}) / F(R)>1000$ and $\mathrm{R}-\mathrm{K}>4.5$, a minority of the full MIPS source population. For this reason, they computed neither the AGN volume densities nor AGN fractions with respect to the full CDFS MIPS galaxy sample. Here, we apply the F08 method to the C-COSMOS field and extend their analysis to the full MIPS source population. This allows us to compute proper AGN volume densities and AGN fractions with respect to the full MIPS source population.

Figure 1 (left panel) compares the $5.8 \mu \mathrm{m}$ luminosities and redshifts of the CDFS (green) and C-COSMOS (yellow) MIPS-selected sources not directly detected in X-rays and with $F(24 \mu \mathrm{m}) / F(R)>1000$ and $\mathrm{R}-\mathrm{K}>4.5$ to those of the X-ray detected population (red and blue symbols for the C-COSMOS and CDFS fields, respectively). Monochromatic luminosities were computed using a linear interpolation between the observed $8 \mu \mathrm{m}$ and $24 \mu \mathrm{m}$ fluxes at the wavelength corresponding to $5.8 \mu \mathrm{m}$ at the source rest frame.

Both MIPS-selected and X-ray-selected COSMOS sources have luminosities $\sim 10$ times higher than the CDFS sources at the same redshifts, as expected since luminous QSOs are rarer than low luminosity AGNs, and the C-COSMOS survey covers an area about 10 times larger than the CDFS at a 10 times brighter $24 \mu \mathrm{m}$ flux limit $(\sim 550 \mu \mathrm{Jy}$ and $40 \mu \mathrm{Jy}$ for CCOSMOS and CDFS, respectively). 

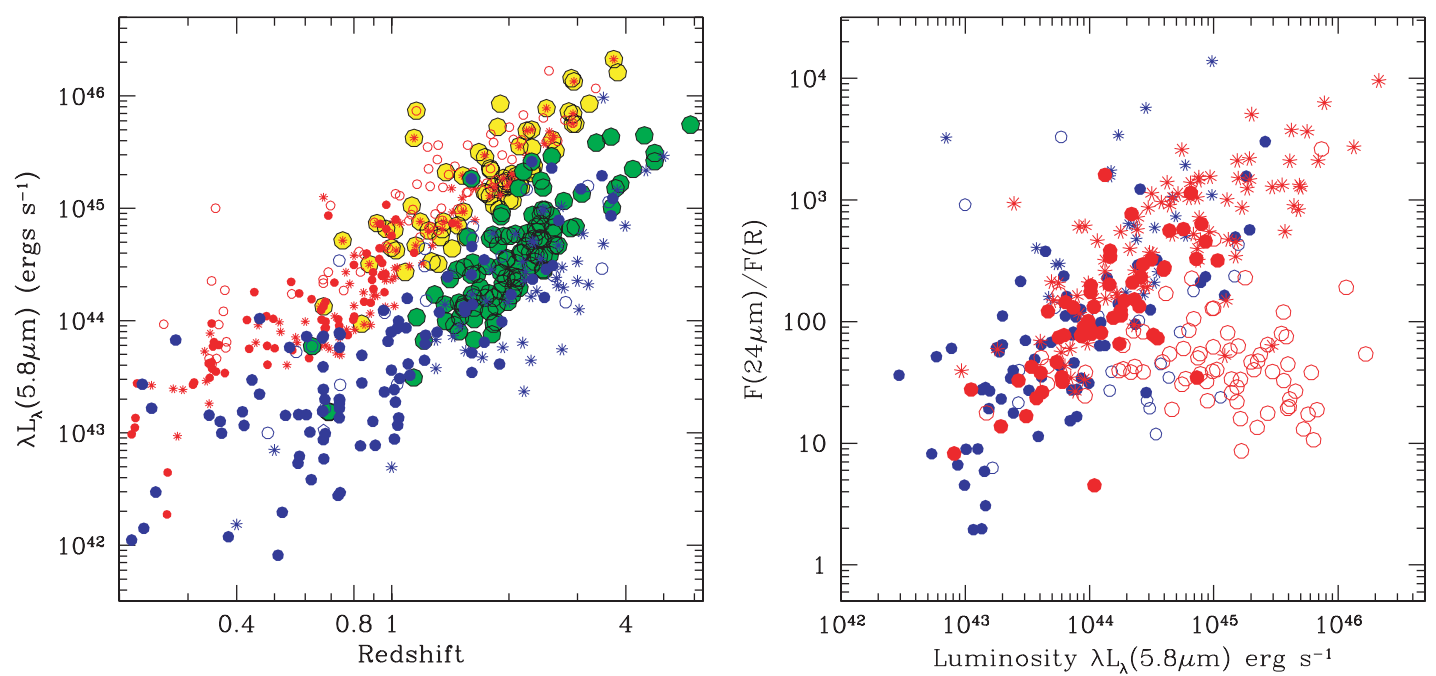

Figure 1. Left panel: the redshift-infrared luminosity, $\log \left(\lambda L_{\lambda}(5.8 \mu \mathrm{m})\right.$ ), plane for the C-COSMOS sources (red and yellow points) and the CDFS sources (blue and green points). Red and blue circles are sources directly detected in the X-rays. Yellow and green points are $24 \mu \mathrm{m}$, non X-ray detected sources with $F(24 \mu \mathrm{m}) / F(R)>1000$, and $R-K>4.5$. Right panel: $F(24 \mu \mathrm{m}) / F(R)$ as a function of the $5.8 \mu \mathrm{m}$ luminosity for two X-ray source samples (GOODS-MUSIC, blue symbols, C-COSMOS, red symbols). In both panels, open circles correspond to spectroscopic type 1 AGNs, filled circles to non type 1 AGNs, and asterisks to objects with photometric redshifts. Note that $F(24 \mu \mathrm{m}) / F(R)$ of nonbroad line AGNs is strongly correlated with the luminosity at $5.8 \mu \mathrm{m}$.

(A color version of this figure is available in the online journal.)

Figure 1 (right panel) shows $F(24 \mu \mathrm{m}) / F(R)$ as a function of the $5.8 \mu \mathrm{m}$ rest frame luminosity $\lambda L_{\lambda}(5.8 \mu \mathrm{m})$ for the C-COSMOS and CDFS X-ray sources. Unobscured AGNs (open symbols in Figure 1) have $F(24 \mu \mathrm{m}) / F(R)$ in the range 10-200, uncorrelated with $\lambda L_{\lambda}(5.8 \mu \mathrm{m})$, as expected because the nuclear emission dominates both optical and midinfrared wavelengths. Obscured AGNs (filled symbols) have $F(24 \mu \mathrm{m}) / F(R)$ spanning a broader range, and correlated with $\lambda L_{\lambda}(5.8 \mu \mathrm{m})$. For a flux-limited sample, the luminosity is strongly correlated with the redshift (Figure 1, left panel). Therefore, sources with high $F(24 \mu \mathrm{m}) / F(R)$ (and high $5.8 \mu \mathrm{m} \mathrm{lu}-$ minosity) have also redshift systematically higher than sources with lower $F(24 \mu \mathrm{m}) / F(R)$.

Figure 2 shows the distribution of $F(24 \mu \mathrm{m}) / F(R)$ as a function of the R-K color. $F(24 \mu \mathrm{m}) / F(R)$ of X-ray selected obscured AGN is correlated with the R-K color, as found by F08 for the CDFS X-ray sources. The isodensity contours of the $24 \mu \mathrm{m}$-selected sources (green curves) follow roughly the same correlation. Unlike the $24 \mu \mathrm{m}$-selected GOODS-MUSIC sources, we do not find a bimodal distribution in R-K colors for the COSMOS sources at high $F(24 \mu \mathrm{m}) / F(R)$ values (see Figure 3, right panel in F08). This is probably due to the higher COSMOS flux limits. The faint, blue, star-forming galaxies found in the GOOD-MUSIC sample are not common in the COSMOS bright MIPS sample.

CT AGNs can also have values of the $F(24 \mu \mathrm{m}) / F(R)$ and $\mathrm{R}-\mathrm{K}$ colors smaller than those adopted by F08, although their fraction to the full infrared-selected population is probably small. To properly account for these sources, we extend the F08 approach by analyzing the full $F(24 \mu \mathrm{m}) / F(R)-\mathrm{R}-\mathrm{K}$ diagram. We divided this plane in nine cells (see Figure 2). The boundaries of the cells were chosen according to the following criteria: (1) cover most of the $F(24 \mu \mathrm{m}) / F(R)-\mathrm{R}-\mathrm{K}$ plane; (2) sample regions not too big in this plane; (3) but at the same time regions containing a number of sources big enough to be statistically meaningful. These nine cells contain most of the COSMOS bright MIPS sample (87\%). Our goal is to estimate the fraction of CT AGNs to the total MIPS source population in each of these cells. Tables 3 and 4 give the number of MIPS-

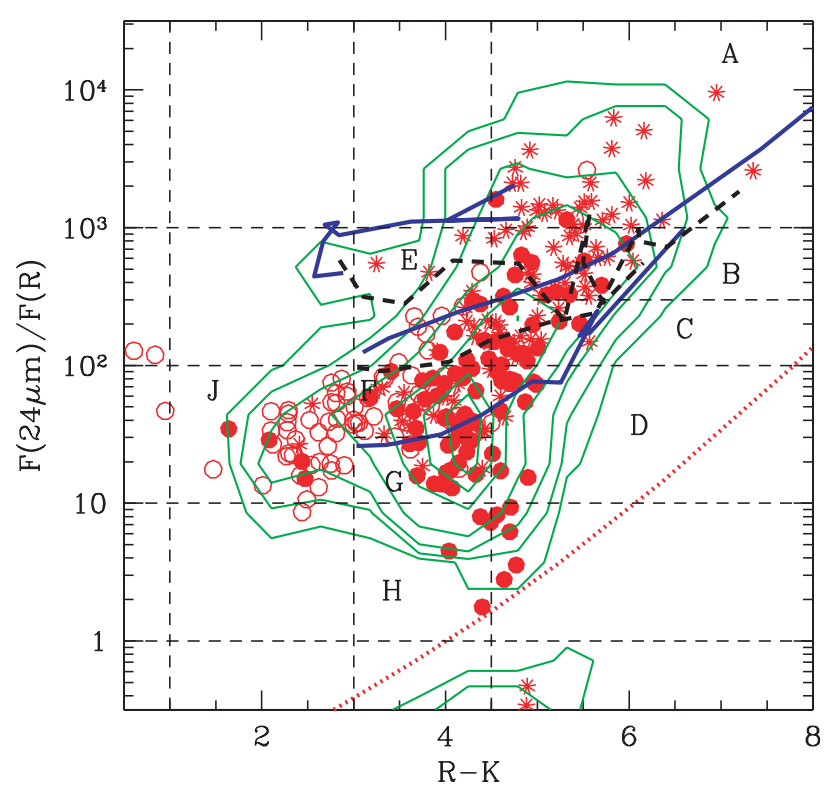

Figure 2. $F(24 \mu \mathrm{m}) / F(R)$ as a function of $\mathrm{R}-\mathrm{K}$ color for the $\mathrm{X}$-ray detected population with a luminosity $>10^{38} \mathrm{erg} \mathrm{s}^{-1}$. Open circles = type 1 AGNs; filled circles $=$ non type 1 AGNs; asterisks $=$ photometric redshifts. Isodensity contours of all COSMOS $24 \mu \mathrm{m}$ sources are overlaid to the plot. Expected evolution from $z=0$ to $z=5$ for three obscured AGNs (blue continuous lines), two star burst (black dashed lines), and a passive elliptical galaxy (red dotted line) are shown for reference (see F08 for details on the SEDs used). The nine cells defined in the $F(24 \mu \mathrm{m}) / F(R)$ plane for the stacking analysis are labeled with letters from A to $\mathrm{J}$ (see Section 2.2 for details).

(A color version of this figure is available in the online journal.)

selected sources in each cell, along with the median redshift and luminosity (with their interquartile ranges) of both MIPS sources with (Table 3) and without (Table 4) a direct X-ray detection.

\section{X-RAY PROPERTIES OF THE $24 \mu \mathrm{M}$-SELECTED SOURCES}

\subsection{Sources with a Direct $X$-ray Detection}

A total of 232 sources of the COSMOS bright MIPS sample are present in the C-COSMOS and XMM-COSMOS cat- 


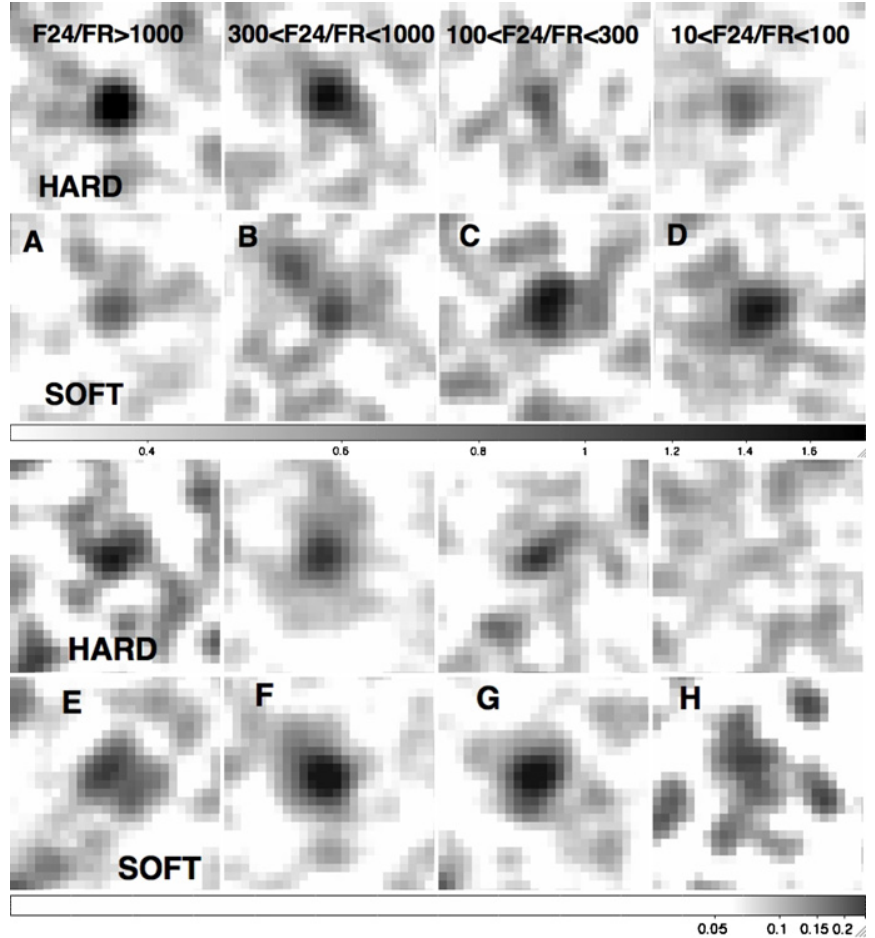

Figure 3. Stacked Chandra images in the hard $1.5-6 \mathrm{keV}$ and soft 0.3 $1.5 \mathrm{keV}$ bands of COSMOS MIPS sources not directly detected in X-rays in eight $F(24 \mu \mathrm{m}) / F(R)$ cells (A, B, C, D, E, F, G, and H). Images have sides of $12 \operatorname{arcsec}$ and have been smoothed with a Gaussian with $1.5 \operatorname{arcsec} \sigma$.

alogs (Elvis et al. 2009; F. Civano et al. 2009, in preparation; Puccetti et al. 2009, in preparation; Brusa et al. 2007; M. Brusa et al. 2009, in preparation). In addition to these sources, we also consider as "detections" 47 sources with more than 10 background-subtracted Chandra counts in the full $0.5-7 \mathrm{keV}$ band, within 5 arcsec of the position of the MIPS source but not present in the C-COSMOS and $X M M$-COSMOS catalogs. This allows us to identify: (1) faint $\mathrm{X}$-ray sources (given the average background, 10 backgroundsubtracted counts in a 5 arcsec radius area correspond to a probability $\sim 5 \times 10^{-3}$ that the detected counts are due to a background fluctuation); (2) MIPS sources with a nearby X-ray source; and (3) MIPS sources found in X-ray groups and clusters of galaxies. The total number of MIPS sources with an X-ray counterpart is $279(\sim 30 \%$ of the full MIPS sample, 254 sources in the nine cells defined in Figure 2). Eleven sources do not have K-band detections, while the remaining 14 sources are scattered in the diagram outside the considered cells $(\mathrm{R}-\mathrm{K}<1$ and $F(24 \mu \mathrm{m}) / F(R)<10)$. Table 3 gives the fraction of MIPS sources with a direct Chandra and/or XMM-Newton detection in the nine $F(24 \mu \mathrm{m}) / F(R)$ and $\mathrm{R}-\mathrm{K}$ cells. The fraction of MIPS sources with a direct $\mathrm{X}$-ray detection is minimum $(\sim 13 \%)$ in cell $\mathrm{G}$, while it is maximum $(79 \%)$ in cell $\mathrm{J}$. Most of the X-ray sources in the latter cell have been spectroscopically identified $(95 \%)$ and the majority (90\%) turned out to be type 1 QSOs, as expected from their colors.

Table 3 gives the median and interquartile range of the X-ray and infrared luminosities of the MIPS sources with X-ray detection in the nine cells. The X-ray luminosity is computed in the rest frame $2-10 \mathrm{keV}$ band to ease the comparison with previous studies. It is computed from the observed $0.5-7 \mathrm{keV}$ flux, assuming a power-law spectrum $F(E) \propto E^{\alpha_{\mathrm{E}}}$ with energy index $\alpha_{E}=0.8$ and Galactic $\mathrm{N}_{H}$. It is not corrected for absorption, and therefore it should be considered a lower limit to the intrinsic luminosity. The log ratio between the observed $5.8 \mu \mathrm{m}$ and $2-10 \mathrm{keV}$ median luminosities of the 35 type 1 AGNs in cell $\mathrm{J}$ is 1.15 . This can be considered to be little affected by absorption, and therefore representative of the ratio between the intrinsic AGN luminosities. The highest luminosity log ratio is for the objects in bin A. Its value (1.7) is significantly higher than in bin $\mathbf{J}$, thus suggesting some obscuration or intrinsically low X-ray emission in the X-ray counterparts of the MIPS sources in this cell.

Table 3 also gives the median and interquartile of the hardness ratios $(H-S) /(H+S)$ computed, using the source counts detected in the $0.3-1.5 \mathrm{keV}(\mathrm{S})$ and $1.5-6 \mathrm{keV}(\mathrm{H})$ bands. The hardness ratio expected for a power-law spectrum with $\alpha_{E}=0.8$ and no absorption in addition to the Galactic one is -0.1 . Values between 0.2 and 0.8 imply absorbing column densities between $10^{22}$ and $10^{23} \mathrm{~cm}^{-2}$ (i.e., Compton-thin absorbers) at typical redshifts of 1-2. Note that the hardness ratio of X-ray sources with $\mathrm{R}-\mathrm{K}>4.5$ is systematically higher than that of bluer sources. Similar results were found by Mignoli et al. (2004); Brusa et al. (2005); and Mainieri et al. (2007). Indeed, the sample of $\mathrm{R}-\mathrm{K}>4.5$ sources is dominated by narrow line AGNs (only 1 broad line AGN out of 43 objects with narrow line or absorption line optical spectra, 30 of which with $\log \mathrm{L}(2-10 \mathrm{keV})>42 \mathrm{erg}$ $\mathrm{s}^{-1}$, and therefore likely to host an active nucleus). Conversely, 69 of the 122 objects with $\mathrm{R}-\mathrm{K}<4.5$ and $\log \mathrm{L}(2-10 \mathrm{keV})>$ $42 \mathrm{erg} \mathrm{s}^{-1}$ are broad line AGNs.

\subsection{X-ray Stacking Analysis of the Sources without a Direct $X$-ray Detection}

The total number of COSMOS bright MIPS sources without a direct X-ray detection is 640. Twenty-three sources are bright stars, while 610 sources have either a spectroscopic or a photometric redshift (224 and 386, respectively). Finally, seven sources are either strongly blended (4) or too faint in any optical band; in both cases the photometry is poor and unreliable and photometric redshifts are not available. Table 4 gives the number of sources without a direct X-ray detection in each of the nine $F(24 \mu \mathrm{m}) / F(R)$ and R$\mathrm{K}$ cells along with their median infrared luminosities and interquartile ranges. The median redshifts and $5.8 \mu \mathrm{m}$ luminosities of the MIPS sources without a direct X-ray detection in all cells but $\mathbf{J}$ are similar to those with $\mathrm{X}$-ray detection. This suggests that most, if not all, QSOs in cell $\mathrm{J}$ have been detected in X-rays, and that the remaining sources are either inactive galaxies or very faint AGNs.

To gain information on the X-ray properties of the MIPS sources without a direct X-ray detection, we performed a detailed stacking analysis of the Chandra counts at the position of all MIPS source for the samples in the nine $F(24 \mu \mathrm{m}) / F(R)$ and $\mathrm{R}-\mathrm{K}$ cells. Indeed, the Chandra deep and uniform coverage allows us to increase the sensitivity (by factors of $\times$ 10-100), using the stacking technique (Daddi et al. 2007; Steffen et al. 2007, F08). Stacking analysis was performed using both the web-based tool for stacking analysis of Chandra data prepared by T. Miyaji (http://saturn.phys.cmu.edu/cstack/) and software developed at "ASDC" by S. Puccetti. Results from the two software in the standard bands 0.5 $2 \mathrm{keV}$ and 2-8 keV were fully consistent for all nine samples. Stacking was performed on single ACIS-I exposures and on the combined mosaic. The results were again fully consistent.

Errors on stacked net source counts and count rates are computed by using both Poisson statistics and a "bootstrap" method (by resampling the objects in the input source list). A 
total of 500 bootstrapped stacked count rates are generated for each source list. Poisson errors turned out to be smaller than the bootstrap error by $3 \%-5 \%$. In the following analysis, we conservatively increase the Poisson error by $10 \%$ to account for other possible systematic errors.

Stacking was performed in two energy bands to allow the evaluation of a hardness ratio. The signal to noise at high X-ray energies is limited by the internal background, whose spectrum is nearly constant as a function of the energy. Indeed, the $2-8 \mathrm{keV}$ band has an internal background $\sim 4$ times higher than the $0.5-2 \mathrm{keV}$ band. To optimize the analysis at high energies, we performed the stacking in several different energy bands and chose the band that gives the highest signal-to-noise ratio for most of the nine samples. The $1.5-6 \mathrm{keV}$ band gave the best results in terms of signal to noise. In the following, we present hardness ratio computed by using this band and the $0.3-$ $1.5 \mathrm{keV}$ band. We performed similar analysis using the 1.5$4 \mathrm{keV}$ and $1.5-5 \mathrm{keV}$ bands as the higher energy band always gives qualitatively similar results.

Source extraction regions were also chosen to optimize the signal-to-noise ratio. We adopted a box with 5 arcsec side (100 ACIS-I square pixels area). A slightly lower signal-to-noise ratio is obtained using boxes with 3 and 6 arcsec side.

Stacked counts in both energy bands are given in Table 4. Detections with a signal to noise higher than four are obtained in all cells but cells $\mathrm{H}$ and J. Figure 3 shows the stacked images of the sources in all cell but $\mathrm{J}$ and in the two energy bands, while Table 4 and Figure 4 show the hardness ratio $(H-S) /(H+S)$ derived from the stacking analysis as a function of $F(24 \mu \mathrm{m}) / F(R)$ for the nine cells. The samples with the highest $F(24 \mu \mathrm{m}) / F(R)$ and red colors (cells A and B) also have the hardest $\mathrm{X}$-ray hardness ratios. A common concern in stacking analyses is that the results may not be representative of population properties, if they are biased by one or a few sources in the stack. We detect in the stack of the sources in cells A and B 59 and 54 background subtracted counts in the $1.5-6 \mathrm{keV}$ band, respectively. Sources can enter in the stack only if they give less than 10 counts in a region of 5 arcsec radius around the MIPS position in the full $0.5-7 \mathrm{keV}$ band. Sources with an higher number of counts are excluded. Given this threshold, the stacked counts in cells A and B must be produced by at least six sources, and probably by many more, since it would be highly unlikely to have six sources near the chosen threshold and all the rest with zero counts.

The hardness ratios of the stacks of the sources without a direct X-ray detection in cells A and B are similar to the median hardness ratios of the X-ray detected sources in the same cells (Table 3). Taken at face values, these hardness ratios can be explained by a power-law spectra with energy index $\sim-0.5$ and $\sim 0$, respectively, reduced at low energy by Galactic absorption only. Since neither AGNs nor known star-forming galaxies have such hard emission spectrum, the observed hardness ratio strongly suggests significant rest frame obscuration. However, for the stacked samples it is not easy to convert a hardness ratio in a typical column density. A direct conversion could produce contradictory results because the X-ray spectrum of the MIPS sources is likely to be more complex than a simple obscured power law. For example, a scattering component can dominate the soft X-ray counts. Furthermore, samples corresponding to different cells have different average redshifts, and therefore comparing hardness ratios is not straightforward, as they are biased toward measuring "softer" HRs for an obscured AGNs at high redshift. To investigate further these issues, we performed

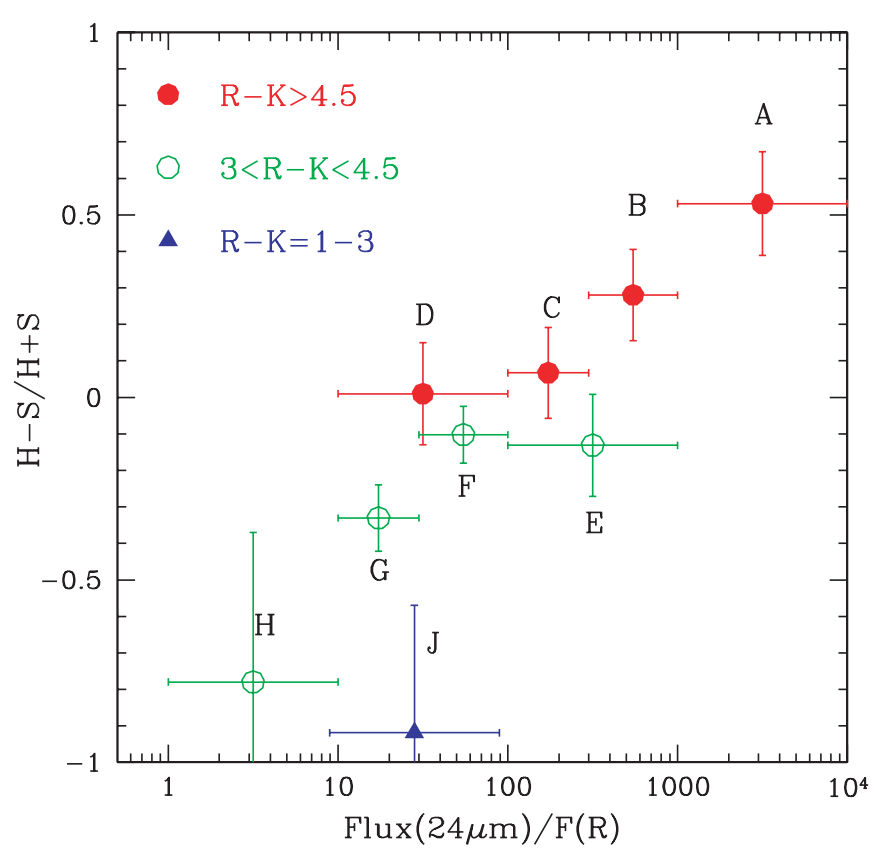

Figure 4. Average hardness ratio $(H-S) /(H+S)$ as a function of $F(24 \mu \mathrm{m}) / F(R)$ for the sources without direct X-ray detection. Filled circle $=$ cells $\mathrm{A}, \mathrm{B}, \mathrm{C}, \mathrm{D}$; open circles $=$ cells $\mathrm{E}, \mathrm{F}, \mathrm{G}, \mathrm{H}$; filled triangle $=$ cell $\mathrm{J}$.

(A color version of this figure is available in the online journal.)

detailed Monte Carlo simulations as described in the following section.

\subsection{Simulations to Assess the Fraction of Obscured AGNs in the 24 um Source Samples}

Following F08, we used the observed flux in the stacked images, together with the hardness ratio $H-S / H+S$, to constrain the fraction of CT AGNs in the MIPS samples. For the MIPS sources in each of the nine $F(24 \mu \mathrm{m}) / F(R)$ and $\mathrm{R}-\mathrm{K}$ cells, we generated simulated $\mathrm{X}$-ray count rates and hardness ratios as a function of the fraction of obscured AGNs to the total MIPS source population in each cell, assuming that the sources without an X-ray detection are either obscured AGNs or star-forming galaxies. We started from the observed redshift and infrared luminosities. For the AGNs, we assumed the $\log \left(\lambda L_{\lambda}(5.8 \mu \mathrm{m}) / L(2-10 \mathrm{keV})\right)$ luminosity ratio given by the following relationship to obtain unobscured $2-10 \mathrm{keV}$ luminosities:

$$
\begin{gathered}
\log \mathrm{L}(2-10 \mathrm{keV})=43.574+0.72(\log \mathrm{L}(5.8 \mu \mathrm{m})-44.2) \\
\text { if } \log \mathrm{L}(5.8 \mu \mathrm{m})>43.04 \\
\log \mathrm{L}(2-10 \mathrm{keV})=\log \mathrm{L}(5.8 \mu \mathrm{m})-0.3 \\
\text { if } \log \mathrm{L}(5.8 \mu \mathrm{m})<43.04 .
\end{gathered}
$$

This has been calibrated using the type 1 AGNs in the CDFS (Brusa et al. 2008) and C-COSMOS (F. Civano et al. 2008, in preparation) fields as shown in Figure 5. The derived relation assumes that the $2-10 \mathrm{keV}$ luminosity, computed directly from the observed fluxes without any correction for intervening absorption, can be considered representative of the intrinsic $\mathrm{X}$-ray luminosity. This is a good approximation for most of the points. However, seven outliers (one in the CDFS sample and six in the C-COSMOS sample) show a low X-ray luminosity, given their infrared luminosity, suggestive of significant 


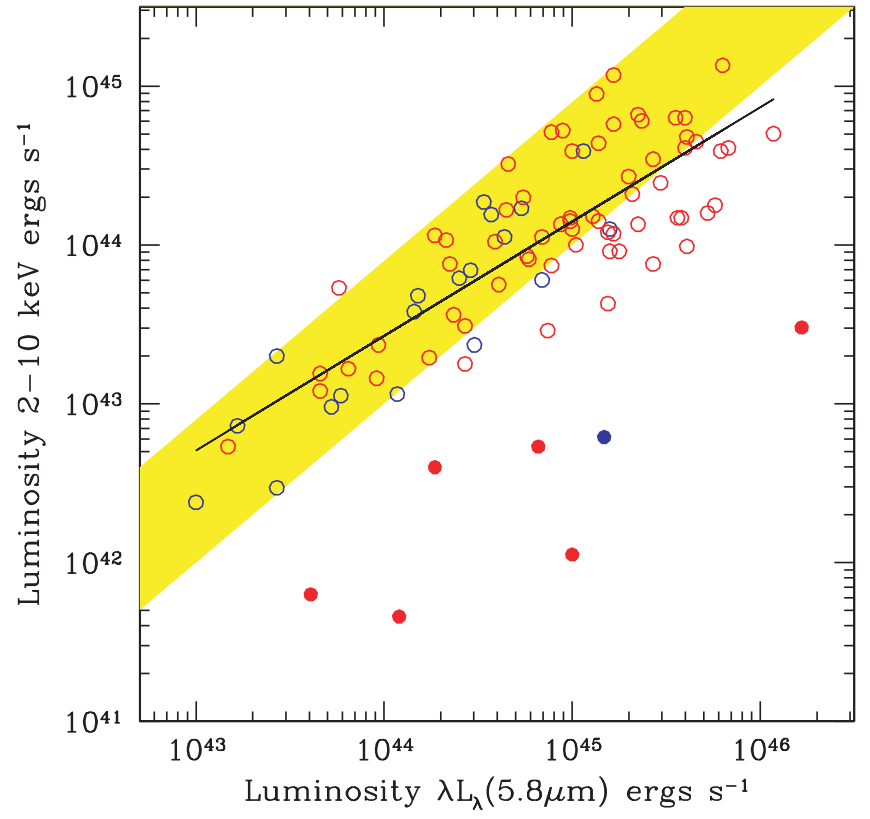

Figure 5. 2-10 keV luminosity (not corrected for obscuration) as a function of the $5.8 \mu \mathrm{m}$ luminosity for two samples of type 1 AGNs: C-COSMOS (red symbols) and CDFS (blue symbols). For most of the points this $2-10 \mathrm{keV}$ luminosity can be considered representative of the intrinsic X-ray luminosity. Filled circles identify seven outliers with small X-ray luminosity with respect to their infrared luminosity, suggesting X-ray absorption in these sources, and therefore that the intrinsic X-ray luminosity is likely underestimated in these cases. For this reason, these sources have been excluded from the analysis. The black solid line is the best fit linear regression in Equation (1). The shaded region is the linear extrapolation of the intrinsic X-ray-to-mid-infrared luminosity ratio found in the local Universe (Lutz et al. 2004).

(A color version of this figure is available in the online journal.)

X-ray obscuration in these sources. These seven points were therefore excluded from the analysis. The $\log \mathrm{L}(2-10 \mathrm{keV})-$ $\log \left(\lambda L_{\lambda}(5.8 \mu \mathrm{m})\right.$ linear regression coefficient in Equation (1) turns out to be very similar to that found by Steffen et al. (2006, 0.643). The relationship in Equation (1) is consistent with the $\log \left(\lambda L_{\lambda}(5.8 \mu \mathrm{m}) / L(2-10 \mathrm{keV})\right)$ luminosity ratio of the highly obscured AGNs in F08 and Silva et al. (2004). In particular, it is well consistent with the ratio found for the type 2 QSO IRAS09104+4109 (Piconcelli et al. 2007, 2009, in preparation). For the star-forming galaxies, we assumed a $\log \left(\lambda L_{\lambda}(5.8 \mu \mathrm{m}) / L(2-10 \mathrm{keV})\right)$ luminosity ratio of 2.38 with a Gaussian dispersion of 0.2 (see Ranalli et al. 2003).

We assumed that the star-forming galaxies are not obscured in X-rays, while the AGNs are highly obscured. We used the Gilli et al. (2007) $\mathrm{N}_{H}$ distribution. This distribution is rapidly increasing toward high column densities; it peaks at about $10^{23.5}$ and then slowly decreases in the Compton-thick regime. The shape is obtained by requiring a simultaneous fit of both the $\mathrm{X}$-ray background spectrum and the source counts in different energy bands $(0.5-2 \mathrm{keV}, 2-10 \mathrm{keV}$, and 5-10 keV). We randomly chose an $\mathrm{N}_{H}$ from the Gilli et al. distribution for each MIPS source. For column densities $\gtrsim 3 \times 10^{24} \mathrm{~cm}^{-2}$, we assumed that the direct emission is completely blocked by photoelectric absorption and Compton scattering. We used power-law spectra with an energy index equal to 0.8 for the AGNs and 0.9 for the star-forming galaxies. In both cases, we assumed a Gaussian dispersion with $\sigma=0.2$ (Ranalli et al. 2003). For the AGNs, we also assumed a Compton reflection component with the same normalization and energy index of the power-law component, assuming an inclination of the reflecting material to the line of sight of 60 degrees, and a scattering component with the same spectral index of the power-law component and normalization $1 / 100$ of the power-law component. This is a conservative value which accounts for recent results on highly obscured AGNs with very small low energy scattering component (Ueda et al. 2007; Comastri et al. 2007). Fluxes in the 0.3-1.5 keV and 1.5$6 \mathrm{keV}$ band were computed by using the unobscured 2$10 \mathrm{keV}$ luminosities and the assumed spectrum. Finally, count rates were computed by using the Chandra on axis response.

We run 12 series of simulations (12 different realizations for each of the sources in each of the nine cells), varying the fraction of AGNs between 0 and $100 \%$ of the MIPS sources in each cell. This fraction is then evaluated in each cell by comparing the output of the simulation with the results of the stacking analysis. Since this analysis was performed excluding the sources directly detected in X-rays, simulations providing more than 10 counts in the $0.3-6 \mathrm{keV}$ band were also excluded from the analysis. Most $(>95 \%)$ of the simulated AGNs with column densities $\lesssim 10^{24} \mathrm{~cm}^{-2}$ turned out to have more than 10 counts in the $0.3-6 \mathrm{keV}$ band, and were excluded from the analysis. This confirms that most unobscured and Compton-thin AGNs would have been detected directly by Chandra. On the other hand, simulations with $\mathrm{N}_{H}>10^{24} \mathrm{~cm}^{-2}$ produced more than 10 counts $\sim 10 \%-20 \%$ of the times in cells A and B. This suggests that some of our faint Chandra detections might be Compton-thick AGNs.

Using these simulations, we converted the observed hardness ratios in a fraction of obscured, most of them CT AGNs to the total MIPS source population in each of the nine cells. Figure 6 (left panel) shows the calibration plot for cell A as an example. Figure 6 (right panel) shows the derived fraction of obscured AGNs to the total MIPS source population as a function of $F(24 \mu \mathrm{m}) / F(R)$. These fractions are also given in Table 4. At the end of the procedure, we verified that the number of sources excluded in each cell is roughly similar to the number of sources actually detected in the real images.

We studied how the derived fractions vary by changing some of the assumptions made to build the simulations. The results turned out qualitatively similar to those reported in Figure 6 and Table 4 in all cases. We first studied the impact on the results of a higher count threshold to exclude entries from the simulations. Setting the threshold at 20 counts rather than 10 does not change the fraction of obscured AGNs in cells A and B, while it slightly reduces this fraction in the other cells.

Assuming a flat $\mathrm{N}_{H}$ distribution with a cut-off at $\mathrm{N}_{H}=$ $10^{26} \mathrm{~cm}^{-2}$ instead of the Gilli et al. (2007) distribution slightly decreases the predicted hardness ratios, thus increasing the fractions of obscured AGNs by 5\%-10\%. The reason is that this flat distribution has a fraction of objects in the $\log \mathrm{N}_{H}$ bin 25-26 higher than the Gilli et al. distribution.

Assuming a fixed conversion factor between the infrared and X-ray luminosities equal to that given by Equation (1) at $\log \mathrm{L}(2-10 \mathrm{keV})=44$ (6.3) does not significantly change the predicted hardness ratios. Assuming a fixed factor twice the previous feature, as if additional components in addition to the nuclear one contribute significantly to the $5.8 \mu \mathrm{m}$ luminosity, slightly increases the predicted hardness ratios, decreasing the fraction of obscured AGNs needed to reproduce the observed ratios by $\sim 5 \%$.

The main parameter affecting the predicted hardness ratio, in addition to the fraction of highly obscured AGNs, is the 

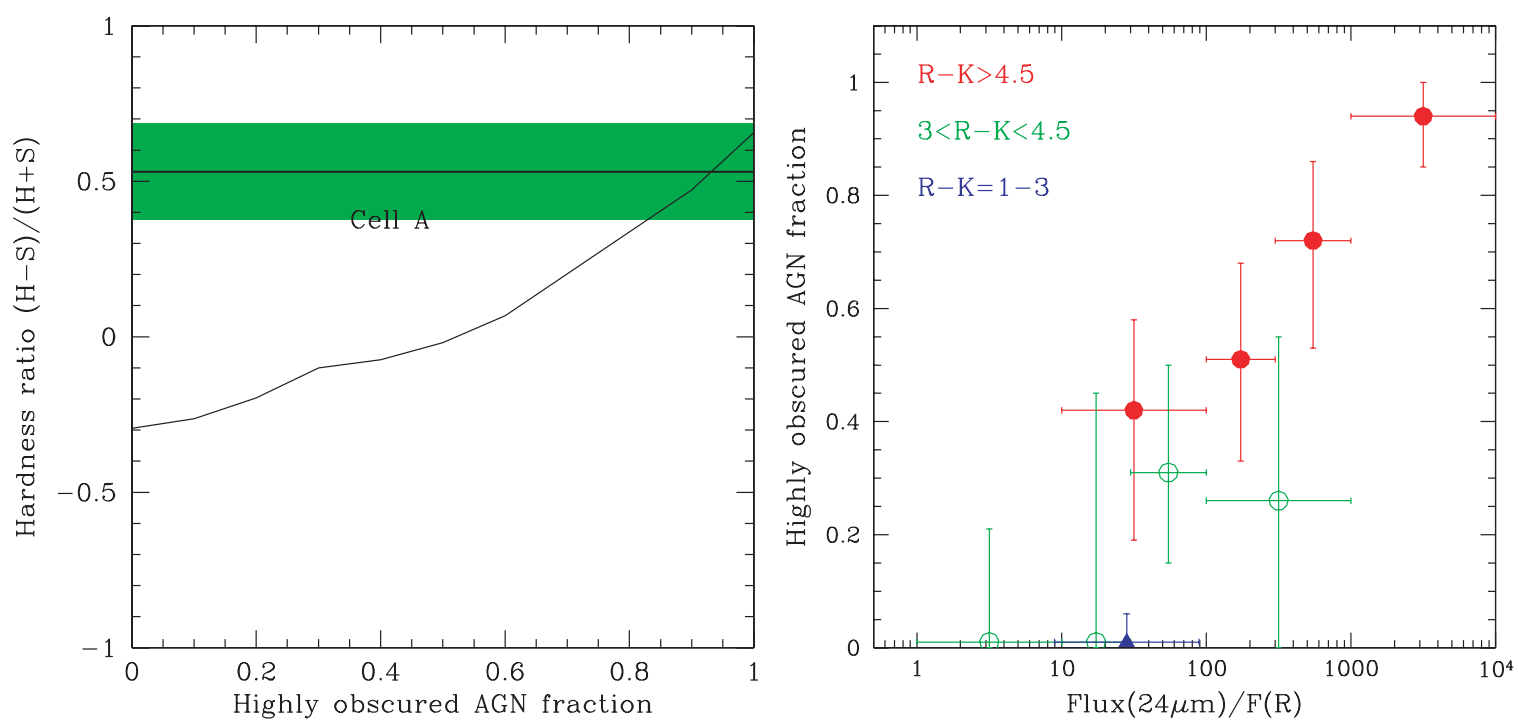

Figure 6. Left panel: the hardness ratio $(H-S) /(H+S)$ as a function of the fraction of CT AGNs in the sample of MIPS sources with a spectroscopic or photometric redshift in cell A. The solid curve is the result of Monte Carlo simulations (see text for details); the thick horizontal line is the average hardness ratios measured in cell A. The colored bands mark the hardness ratio statistical uncertainties. Right panel: the fraction of CT AGNs to the total MIPS source population as a function of $F(24 \mu \mathrm{m}) / F(R)$. Filled circle $=$ cells A, B, C, D; open circles $=$ cells E, F, G, H; filled triangle $=$ cell $\mathrm{J}$.

(A color version of this figure is available in the online journal.)

Table 5

Template Fits to the Infrared SEDs of MIPS Sources With a Direct X-ray Detection

\begin{tabular}{lccccccccr}
\hline \hline Template & A & B & C & D & E & F & G & H & J \\
\hline Passive galaxies $^{\mathrm{a}}$ & 0 & 0 & 0 & 0 & 0 & 1 & 0 & 1 & 0 \\
\hline Star-forming galaxies $^{\mathrm{b}}$ & 0 & 4 & 2 & 9 & 6 & 15 & 18 & 3 & 1 \\
\hline Obscured AGNs and QSOs $^{\mathrm{c}}$ & 25 & 20 & 21 & 5 & 15 & 33 & 1 & 0 & 19 \\
\hline Unobscured AGNs and QSOs $^{\mathrm{d}}$ & 6 & 9 & 1 & 0 & 5 & 12 & 0 & 0 & 19 \\
\hline Total & 31 & 34 & 24 & 14 & 26 & 61 & 19 & 4 & 41
\end{tabular}

Notes.

a Elliptical $+\mathrm{S} 0+$ hybrid passive with AGN contribution $<50 \%$.

${ }^{\text {b }}$ Spiral + M82 + Arp220 + N6090 + hybrid with AGN contribution $<50 \%$.

c Seyfert 1.8 + Seyfert 2 + red QSOs + I19254 + Mark231 + A2690_75 + BPM16274_69 + IRAS09104+4109 + NGC6240 + hybrid passive and active with AGN contribution $\geqslant 50 \%$.

d Seyfert $1+$ QSOs.

energy index assumed for the star-forming galaxies. Assuming a $20 \%$ steeper (or flatter) spectral index for these objects increases (or decreases) the fractions of obscured AGNs by $\sim 10 \%$. The hardest hardness ratios are observed in cells $\mathrm{A}$ and $\mathrm{B}$. These ratios can be produced by a power-law spectrum with energy index -0.5 and 0 , respectively reduced at low energy by Galactic absorption only. Should star-forming galaxies at $z \sim 1.9$ and $\sim 1$ (the median redshift of the sources in cells $\mathrm{A}$ and $\mathrm{B}$, respectively) exhibit such extremely hard spectra, they will be able to explain the observed hardness ratios.

\section{SED FITTINGS}

To characterize the infrared SED of the MIPS-selected sources, we fitted the SED with a library of empirical templates (Polletta et al. 2007; Pozzi et al. 2007, F08, Salvato et al. 2008), fixing the redshift to the spectroscopic redshift or, if this is not available, to the photometric redshift. To limit the importance of dust extinction, we limited the analysis to the observed bands with wavelengths $\gtrsim 2 \mu \mathrm{m}$. We used the 25 templates of F08
Table 6

Template Fits to the Infrared SEDs of MIPS Sources Without a Direct X-ray Detection

\begin{tabular}{lccccccccr}
\hline \hline Template & A & B & C & D & E & F & G & H & J \\
\hline Passive galaxies $^{\mathrm{a}}$ & 0 & 2 & 2 & 1 & 0 & 1 & 0 & 0 & 0 \\
\hline Star-forming galaxies $^{\mathrm{b}}$ & 10 & 31 & 16 & 42 & 19 & 66 & 124 & 10 & 1 \\
\hline Obscured AGN and QSOs $^{\mathrm{c}}$ & 29 & 28 & 63 & 8 & 25 & 59 & 0 & 0 & 9 \\
\hline Unobscured AGN and QSOs $^{\mathrm{d}}$ & 3 & 3 & 0 & 0 & 2 & 0 & 0 & 0 & 1 \\
\hline Total & 42 & 64 & 81 & 51 & 46 & 126 & 124 & 10 & 11
\end{tabular}

Notes.

${ }^{\text {a }}$ Elliptical $+\mathrm{S} 0+$ hybrid passive with AGN contribution $<50 \%$.

${ }^{\text {b }}$ Spiral + M82 + Arp220 + N6090 + hybrid with AGN contribution $<50 \%$.

c Seyfert 1.8 + Seyfert 2 + red QSOs + I19254 + Mark231 + A2690_75 + BPM16274_69 + IRAS09104+4109 + NGC6240 + hybrid passive and active with AGN contribution $\geqslant 50 \%$.

d Seyfert $1+$ QSOs.

plus additional templates of hybrid galaxy plus AGN sources by Salvato et al. (2008). Table 5 and 6 give the number of sources best fitted by four broad template categories in each of the nine cells for sources with and without a direct X-ray detection. We counted as obscured AGN sources best fitted by the Salvato et al. (2008) hybrid models with an AGN contribution larger than $50 \%$. Sources best fitted by hybrid templates with a smaller AGN contribution are counted as passive or star-forming galaxies. Note that the hybrid models of Salvato et al. (2008) are normalized around $1 \mu \mathrm{m}$, and therefore templates with an AGN contribution greater than $50 \%$ at this wavelength may still have the bolometric luminosity dominated by star light. We observe that most sources in cell A of both samples are best fitted by obscured AGN templates ( $81 \%$ and $75 \%$, respectively).

Concerning the sample with a direct X-ray detection, the fraction of obscured or unobscured AGNs to the total MIPS source population is dominant in all cells but $\mathrm{G}$ and $\mathrm{D}$, where the majority of best fit templates are those of star-forming galaxies.

The number of best fit unobscured AGN templates among the samples of MIPS sources without a direct 
$\mathrm{X}$-ray detection is small in all cells, as expected, since these objects would be more easily directly detected in X-rays. The fraction of best fit, obscured AGN templates to the total is high in cells $\mathrm{A}$ and $\mathrm{C}$, while the number of best fit, star-forming galaxies templates is high in cells $\mathrm{D}, \mathrm{F}$, and especially in cell $\mathrm{G}$, where the totality of best fit templates are those of star-forming galaxies (Table 6).

In conclusion, the result of the SED fitting is qualitatively consistent with the results obtained from the analysis of the $\mathrm{X}$-ray properties of the MIPS sources presented in the previous sections and summarized in Figure 6.

\section{THE AGN FRACTION}

We can now evaluate the total AGN fraction (including unobscured, moderately obscured, and highly obscured AGNs) in a $24 \mu \mathrm{m}$ source sample. About $75 \%$ of the MIPS sources with a direct Chandra detection have an X-ray (2-10 keV) luminosity $>10^{42} \mathrm{erg}^{-1}$, and are therefore likely to host an AGN. This already makes $\sim 23 \%$ of the full COSMOS MIPS sample. Taken at face values, the infrared-selected CT AGN fractions given in Table 4 would imply that $36 \pm 11 \%$ of the COSMOS MIPS sources without a direct Chandra detection host an AGN ( 26\% of the full MIPS sample) a fraction similar to that of the X-ray detected AGNs. The total fraction of AGNs in the full MIPS sample, obtained by adding the two previous fractions, is $49 \pm 10 \%$. At the typical $24 \mu \mathrm{m}$ fluxes of the COSMOS bright MIPS sample (flux limit $\sim 550 \mu \mathrm{Jy}$, median flux $\sim 750 \mu \mathrm{Jy}$ ), this is quite a large fraction compared with previous studies (see Brand et al. 2006 and references therein). A more accurate comparison with the study of Brand et al. (2006) can be made by considering the sources at $z>0.6$ only (Brand et al. use this redshift cut to avoid contamination from low- $z$ star-forming galaxies). The left panel of Figure 7 shows the AGN fractions in the COSMOS MIPS bright sample at $z>0.6$ for both sources with a direct X-ray detection and CT AGNs selected using infrared/optical colors and the Chandra stacking analysis presented in Sections 3.2 and 3.3. The fraction of MIPS AGNs with a direct X-ray detection is already higher than the Brand et al. (2006) estimates at the same $24 \mu$ m fluxes. The fraction of AGNs with X-ray luminosity higher than $10^{43}$ erg $\mathrm{s}^{-1}$ is consistent with the Brand et al.'s estimates. The fraction of X-ray AGNs is also of the same order of magnitude of CT AGNs. Adding the fractions of X-ray selected and CT AGN results in a total fraction of AGNs in the COSMOS bright MIPS sample at $z>0.6$ of $0.67 \pm 0.06$, a factor of 2 higher than the Brand et al. (2006) estimates. The reason for this apparent inconsistency is that while Brand et al. (2006) assume that the AGNs dominates the bolometric luminosity, in many of the sources of our MIPS sample the AGNs the bolometric luminosity is dominated by the host galaxy. This can also be appreciated by considering the right panel of Figure 7. It shows the distribution of the $F(24 \mu \mathrm{m}) / F(8 \mu \mathrm{m})$ flux ratio for several COSMOS MIPS samples at $z>0.6$. X-ray-selected type 1 AGNs peak around $\log (F(24 \mu \mathrm{m}) / F(8 \mu \mathrm{m}))=0$, and their distribution is consistent with that of X-ray detected AGNs in the Bootes field. On the other hand, the distribution of X-ray-selected AGNs with an optical spectrum not showing broad lines (non type 1 AGNs), and that of X-ray AGNs with only a photometric redshift, is significantly shifted toward higher $\log (F(24 \mu \mathrm{m}) / F(8 \mu \mathrm{m})$ values. Note as the latter distribution is similar to the distribution of sources in cell A without a direct X-ray detection. Brand et al. (2006) associate with the AGN population the peak of the MIPS source distribution around $\log (F(24 \mu \mathrm{m}) / F(8 \mu \mathrm{m})=0$, and therefore miss part of the $\mathrm{X}$-ray selected AGNs without broad lines in their optical spectra or without optical spectra, and CT AGNs without a direct $\mathrm{X}$-ray detection with a higher $\log (F(24 \mu \mathrm{m}) / F(8 \mu \mathrm{m})$ ratio. Both populations could be recovered in the COSMOS field, thanks to the much deeper X-ray coverage (a Chandra exposure time 20-40 times longer than that on the Bootes field), which allowed the direct detection of Seyfert 2-like galaxies up to $z \sim 1$ and a detailed stacking analysis of the MIPS sources without a direct $\mathrm{X}$-ray detection.

\section{THE DENSITY OF CT AGNs}

In the previous sections, we estimated the fraction of obscured AGNs to the total MIPS source population, not detected directly in X-rays, but visible in the stacked Chandra images, in each of the nine cells defined in the $F(24 \mu \mathrm{m}) / F(R)-\mathrm{R}-\mathrm{K}$ diagram. Our simulations showed that most of these AGNs are likely to be CT, and therefore we will call them CT AGNs (or CT QSOs, when referring to high luminosity sources) for the sake of simplicity. We can now compute the volume density of the MIPS-selected sources, correcting for this fraction, to obtain the density of CT AGNs in different redshift and luminosity bins. We use for this calculation the standard $1 / \mathrm{V}_{\max }$ method (Schmidt 1968, Lilly et al. 1995; Cowie et al. 2003). While it is well known that this method is not free from biases (the main one is that it does not account for evolution within each $L$ and $z$ bins), it is robust enough to derive general trends (see, e.g., Cowie et al. 2003).

Figure 8 (left panel) shows the infrared luminosity-redshift plane for the MIPS sources without a direct X-ray detection in cells A, B, and C. The loci of four SEDs of highly obscured AGNs computed at the $24 \mu \mathrm{m}$ flux limit of $550 \mu \mathrm{Jy}$ are also showed. Two redshift-luminosity bins chosen for the computation of the CT AGNs volume density are also marked in figure. The two bins have been chosen according to the following three criteria:

1. The bins must lie above the lowest limit for an obscured AGN SED corresponding to our $24 \mu \mathrm{m}$ flux limit. We can therefore consider the source samples in these bins relatively little affected by complex selection effects.

2. The redshift and luminosity ranges should not be too large to avoid strong biases in the calculation of the volume densities as explained above.

3. The bins should be cut to maximize the number of sources included in the analysis at the relevant redshifts to keep statistical errors small.

Figure 8 (left panel) shows that a reasonable compromise is to limit the analysis to the sources in the redshift-luminosity bins given in Table 7 . This table gives for each of these bins the number of MIPS sources without a direct X-ray detection in some of the nine $F(24 \mu \mathrm{m}) / F(R)$ and $\mathrm{R}-\mathrm{K}$ cells (the total number of sources in each cell is given in parentheses in the last column of Table 7).

Because of the strong correlation of $F(24 \mu \mathrm{m}) / F(R)$ (and therefore R-K) with $\log \left(\lambda L_{\lambda}(5.8 \mu \mathrm{m})\right)$, the largest numbers of sources with large infrared luminosity are found in cells A and $\mathrm{B}$, which are also the cells with the highest fraction of CT AGNs. On the other hand, the largest number of sources with intermediate luminosity at $z=0.7-1.2$ are found in cell $\mathrm{C}$. The contribution of sources in cells $\mathrm{E}, \mathrm{F}, \mathrm{G}$, and $\mathrm{H}$ is small in both luminosity-redshift bins. The volume densities of CT AGNs and CT QSOs are computed using the sources 

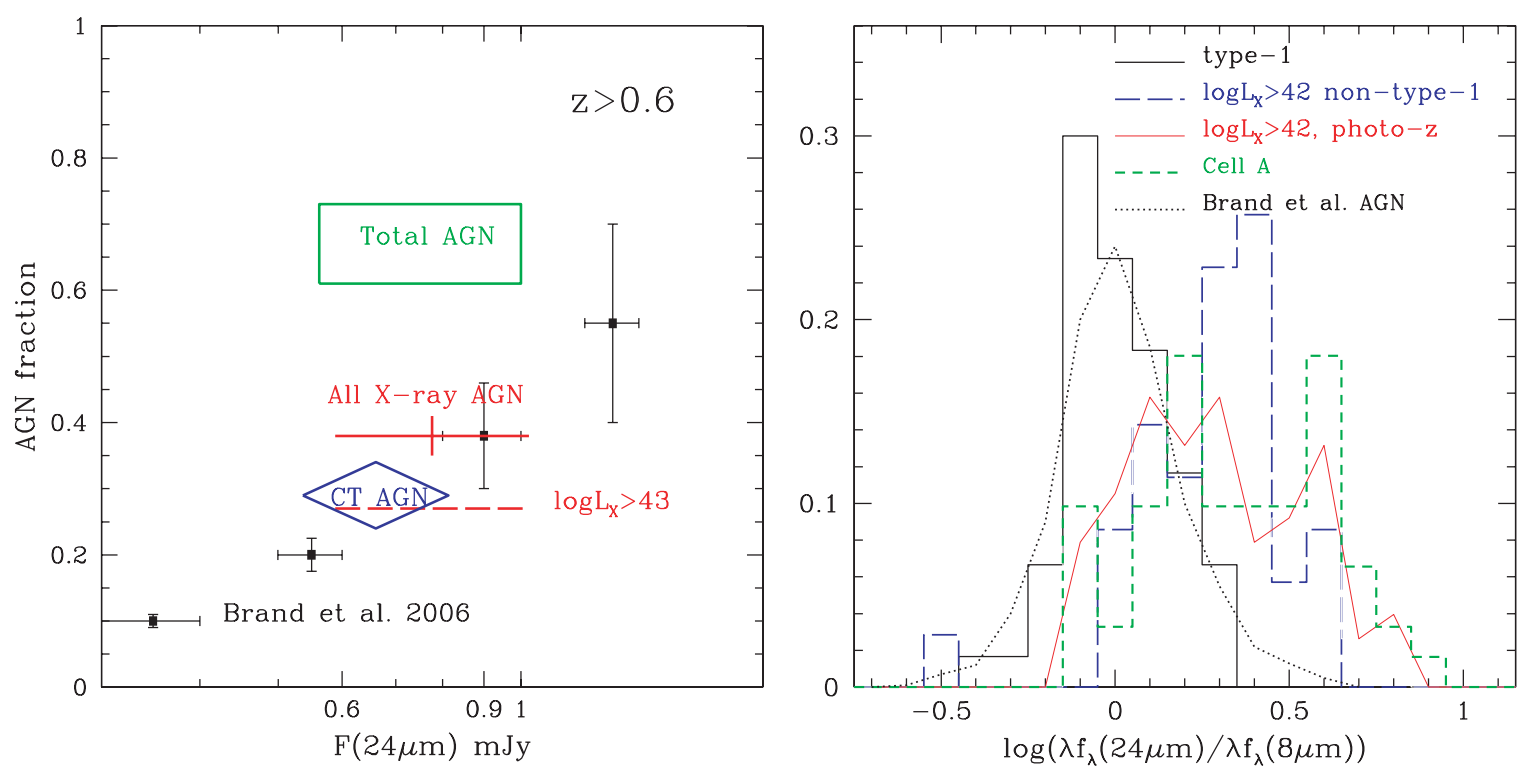

Figure 7. Left panel: the AGN fraction as a function of the $24 \mu \mathrm{m}$ flux for $z>0.6$ sources. Red cross and dashed line $=$ AGNs with a direct X-ray detection; blue diamond $=$ CT AGNs without a direct X-ray detection; green box = total AGN fraction. The black points are the AGN fraction of Brand et al. (2006). Right panel: the distribution of the $F(24 \mu \mathrm{m}) / F(8 \mu \mathrm{m})$ flux ratio for several COSMOS MIPS samples at $z>0.6$ : black, solid histogram $=$ type 1 AGNs with a direct $\mathrm{X}$-ray detection; blue, long-dashed histogram $=$ type 2 AGNs with a direct X-ray detection; red thin solid line $=$ sources with photometric redshift and an X-ray luminosity $>10^{42} \mathrm{erg} \mathrm{s}^{-1}$; and green, short-dashed histogram = sources in cell A without a direct X-ray detection. The Brand et al. (2006) X-Bootes AGNs distribution is showed as a comparison (the black, dotted line).

(A color version of this figure is available in the online journal.)
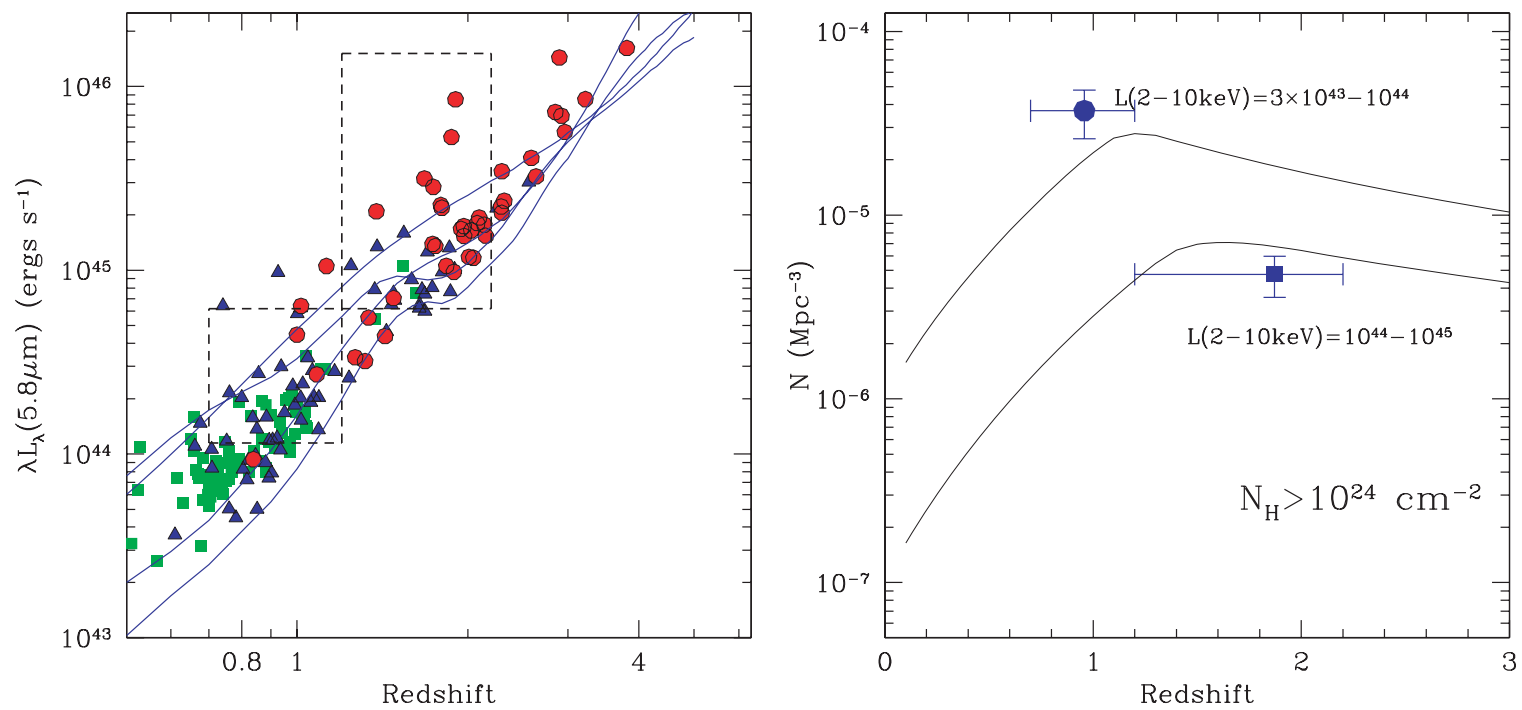

Figure 8. Left panel: the redshift-luminosity plane of the MIPS sources in cell A (red circles), cell B (blue triangles), and cell C (green squares). Two redshift-luminosity bins chosen for the computation of the CT AGN volume density are marked as dashed boxes. The solid curves show the loci of four SEDs of highly obscured AGNs for the $24 \mu \mathrm{m}$ flux limit of $550 \mu \mathrm{Jy}$. Right panel: volume density of COSMOS MIPS-selected CT AGNs. Solid curves are the expectation of the Gilli et al. (2007) model in two luminosity bins.

(A color version of this figure is available in the online journal.)

in Table 7, a $24 \mu \mathrm{m}$ flux limit of $550 \mu \mathrm{Jy}$ and correcting the resulting density of MIPS sources for the fraction of CT AGNs in Table 4. The densities are reported in Table 8 and in Figure 8 (right panel). The two $L(5.8 \mu \mathrm{m})$ luminosity bins in Table 8 correspond to unobscured $2-10 \mathrm{keV}$ luminosities of $3 \times 10^{43}-10^{44}$ and $10^{44}-10^{45} \mathrm{erg} \mathrm{s}^{-1}$ using the $L(5.8 \mu \mathrm{m})-$ $L(2-10 \mathrm{keV})$ luminosity conversion of Equation (1).

\section{DISCUSSION}

We analyzed the X-ray properties of a sample of MIPS $24 \mu \mathrm{m}$ sources with a signal-to-noise ratio $>4$ and a $24 \mu \mathrm{m}$ flux limit of $\sim 550 \mu \mathrm{Jy}$ detected in an area of the COSMOS field with deep Chandra coverage. Of the 919 MIPS sources, 232 have a robust Chandra detection (Elvis et al. 2009). Additional 47 sources have more than 10 background-subtracted Chandra counts within 5 arcsec of the position of the MIPS source, but they are not present in the C-COSMOS and XMM-COSMOS catalogs. These may well be faint X-ray sources just below the adopted detection threshold. The fraction of X-ray detections for the COSMOS bright MIPS sample is therefore between $25 \%$ and $30 \%$. About $75 \%$ of these sources have an X-ray $(2-10 \mathrm{keV})$ luminosity $>10^{42} \mathrm{erg}^{-1}$, and are therefore likely 
Table 7

Number of Sources in Luminosity-Redshift Bins

\begin{tabular}{lccc}
\hline \hline Redshift & $\log \left(\lambda L_{\lambda}(5.8 \mu \mathrm{m})\right)$ & Cell & \# of Sources \\
\hline $1.2-2.2$ & $44.79-46.18$ & A & $21(40)$ \\
$1.2-2.2$ & $44.79-46.18$ & B & $15(64)$ \\
$1.2-2.2$ & $44.79-46.18$ & $\mathrm{C}$ & $2(81)$ \\
$1.2-2.2$ & $44.79-46.18$ & $\mathrm{D}$ & $0(51)$ \\
$1.2-2.2$ & $44.79-46.18$ & $\mathrm{E}+\mathrm{F}+\mathrm{G}+\mathrm{H}$ & $3(306)$ \\
$0.7-1.2$ & $44.06-44.79$ & $\mathrm{~A}$ & $2(40)$ \\
$0.7-1.2$ & $44.06-44.79$ & $\mathrm{~B}$ & $25(64)$ \\
$0.7-1.2$ & $44.06-44.79$ & $\mathrm{C}$ & $29(81)$ \\
$0.7-1.2$ & $44.06-44.79$ & $\mathrm{D}$ & $2(51)$ \\
$0.7-1.2$ & $44.06-44.79$ & $\mathrm{E}+\mathrm{F}+\mathrm{G}+\mathrm{H}$ & $11(306)$ \\
\hline
\end{tabular}

to host an AGN. For the sources not directly detected in the Chandra images, we computed stacked count rates in the 0.3 $1.5 \mathrm{keV}$ and $1.5-6 \mathrm{keV}$ bands. We found a strong correlation between the hardness ratio of the stacked count rates and the $F(24 \mu \mathrm{m}) / F(R)$ flux ratio (and the $\mathrm{R}-\mathrm{K}$ color). We use the stacked count rates and hardness ratios, together with detailed Monte Carlo simulations, to estimate the fraction of Comptonthick AGNs in nine cells defined in the $F(24 \mu \mathrm{m}) / F(R)-\mathrm{R}-\mathrm{K}$ diagram. The simulations were performed assuming that the sources without an X-ray detection are either obscured AGNs or star-forming galaxies. The results, reported in Table 4 and Figure 6, suggest that a large fraction of "IR excess" sources should host an obscured but active nucleus. In particular, the hardness ratios measured in cells A and B, those with the highest $F(24 \mu \mathrm{m}) / F(R)$ flux ratio (Figure 4), would imply a fraction of Compton-thick AGNs as high as 0.93-0.73, respectively. These results were obtained by using reasonable assumptions for the AGNs and star-forming galaxies, X-ray spectra and their normalization to the $5.8 \mu \mathrm{m}$ luminosity. In particular, we adopted for the star-forming galaxies a power-law X-ray spectrum with an energy spectral index 0.9 , similar to that found in star-forming galaxies accessible to detailed X-ray spectroscopy. To explain the observed hardness ratios without resorting to a large population of $\mathrm{CT}$ AGNs would require extremely flat or even inverted energy spectral indices for most star-forming galaxies at $z=1-2$, a spectrum never observed so far in this class of objects at smaller cosmological distances. This would imply a rather extreme cosmological evolution of the $\mathrm{X}$-ray spectrum of star-forming galaxies, which, while cannot be fully excluded, appears nevertheless unlikely. Furthermore, the interpretation of the observed hardness ratio in terms of a high fraction of CT AGNs is fully consistent with the results of template fitting to the observed MIPS source's SEDs.

Recently, Donley et al. (2008) questioned the "IR excess" technique to select highly obscured AGNs, claiming that CDFS "IR excess" samples may be more contaminated by moderately obscured AGNs and star-forming galaxies than estimated by Daddi et al. (2007) and F08. For the purposes of this paper, we limit ourselves to remark that this can hardly be the case for luminous QSOs in cell A. In fact, an unobscured QSO with $L(2-10 \mathrm{keV})=10^{44} \mathrm{erg} \mathrm{s}^{-1}$ at $z=2.2$ (the upper limit of the redshift bin considered in Section 5), would produce between 40 and 80 counts in the C-COSMOS images for the two typical exposure times in Table 2, and it would be easily detected. A QSO with the same redshift and luminosity but with a column density of $5 \times 10^{23} \mathrm{~cm}^{-2}$ would still produce between 10 and 20 counts, thus having a high probability of being detected. Should this source be obscured by a column density as high as $10^{24} \mathrm{~cm}^{-2}$, it would still produce between 6 and 12 counts (direct emission only, without considering the likely contribution from a scattering component), with a nonnegligible probability of being directly detected. We conclude that unobscured or moderately obscured QSOs cannot be present in the sample of MIPS source without a direct X-ray detection at $z=1.2-2.2$ and $\log \mathrm{L}(2-$ $10 \mathrm{keV})=44-45$. This sample may contain star-forming galaxies. However, if this component is the dominant one, then

Table 8

AGN Volume Densities

\begin{tabular}{lccccc}
\hline \hline Redshift & $\begin{array}{c}\log \left(\lambda L_{\lambda}(5.8 \mu \mathrm{m})\right) \\
\mathrm{erg} \mathrm{s}^{-1}\end{array}$ & $\begin{array}{c}\log \mathrm{L}(2-10 \mathrm{keV}) \\
\mathrm{erg} \mathrm{s}^{-1}\end{array}$ & $\begin{array}{c}\text { CT AGN } \\
\mathrm{Mpc}^{-3}\end{array}$ & $\begin{array}{c}\text { Tot. X-ray AGN } \\
\mathrm{Mpc}^{-3}\end{array}$ & $\begin{array}{c}\text { Unobscured X-ray AGNa }^{\mathrm{a}} \\
\mathrm{Mpc}^{-3}\end{array}$ \\
\hline $1.2-2.2$ & $44.79-46.18$ & $44-45$ & $(4.8 \pm 1.1) \times 10^{-6}$ & $1.1 \times 10^{-5}$ & $5.4 \times 10^{-6}$ \\
$0.7-1.2$ & $44.06-44.79$ & $43.477-44$ & $(3.7 \pm 1.1) \times 10^{-5}$ & $5.4 \times 10^{-5}$ & $3.0 \times 10^{-5}$ \\
\hline
\end{tabular}

Note. ${ }^{a}$ Evaluated using the La Franca et al. (2005) luminosity function parameterization.

Table 9

Previous CT AGN Volume Densities Determinations

\begin{tabular}{|c|c|c|c|c|}
\hline Paper & Field & Redshift & $\begin{array}{l}\text { Luminosity } \\
\operatorname{erg~s}^{-1}\end{array}$ & CT AGN density \\
\hline Daddi et al. (2007) & CDFS & $1.4-2.5$ & $\mathrm{~L}(2-10 \mathrm{keV})=(1-4) \times 10^{43}$ & $\approx 2.6 \times 10^{-4} \mathrm{Mpc}^{-3}$ \\
\hline Fiore et al. (2008) & CDFS & $1.2-2.6$ & $\log \mathrm{L}(2-10 \mathrm{keV}) \gtrsim 43$ & $\sim 100 \%$ X-ray-selected AGN \\
\hline Donley et al. (2008) & CDFS & - & - & 54-94\% ${ }^{\text {a }}$ X-ray-selected AGN \\
\hline Alexander et al. (2008) & $\mathrm{CDFN}$ & $2-2.5$ & $\log \mathrm{L}(2-10 \mathrm{keV})=44-45$ & $0.7-2.5 \times 10^{-5} \mathrm{Mpc}^{-3}$ \\
\hline Martinez-Sansigre et al. (2007) & SWIRE SXDS & $1.7-4$ & $\log \mathrm{L}_{\mathrm{bol}} \gtrsim 47$ & $\gtrsim$ unobscured QSOs \\
\hline Polletta et al. (2008) & SWIRE, NDWFS, FLS & $1.3-3$ & $\mathrm{~L}(6 \mu \mathrm{m}) \gtrsim 4 \times 10^{45}$ & $37-65 \%$ total AGN population \\
\hline Della Ceca et al. (2008) & $X M M$ HBS & 0 & $\log \mathrm{L}(2-10 \mathrm{keV})=43$ & $(0.8-2.8) \times 10^{-5} \mathrm{Mpc}^{-3}$ \\
\hline Della Ceca et al. (2008) & $X M M$ HBS & 0 & $\log \mathrm{L}(2-10 \mathrm{keV})=44$ & $(1-5) \times 10^{-7} \mathrm{Mpc}^{-3}$ \\
\hline Della Ceca et al. (2008) & $X M M$ HBS & 0 & $\log \mathrm{L}(2-10 \mathrm{keV})=45$ & $(1-25) \times 10^{-10} \mathrm{Mpc}^{-3}$ \\
\hline
\end{tabular}

Notes.

a The lower limit refers to Spitzer selection only, and the upper limit includes the contribution of AGNs selected because of their high radio to infrared flux ratio.

b The lower limit refers to QSO obscured by a compact torus, the upper limit to the global fraction of obscured QSOs to the total QSO population in that redshift-luminosity bin. 
it would be difficult to explain the high hardness ratio measured for these sources (see above). Similar arguments apply to the sources in the redshift bin 0.7-1.2 and luminosity bin $\log$ L(2$10 \mathrm{keV})=43.477-44$, which are located mainly in cells B and C (see Table 7). We conclude that the "IR excess" selection appears quite robust, at least regarding AGNs with intermediate to high luminosity at $z=0.7-2.2$.

\subsection{The Cosmic Evolution of Obscured AGNs}

The bright flux limit of the COSMOS bright MIPS sample allows a rather limited coverage of the luminosity-redshift plane (see Figure 8, left panel). Nevertheless, we could select two redshift-luminosity bins in which the $24 \mu \mathrm{m}$ source samples can be considered reasonably complete. This allows us to search for cosmic evolution of obscured AGNs, not directly detected in $\mathrm{X}$-rays.

Table 8 gives the volume densities of CT AGNs in the two redshift-luminosity bins. They were calculated by correcting the volume density of the MIPS $24 \mu \mathrm{m}$ source for the fraction of obscured AGNs not directly detected in X-rays given in Table 4. It should be noted that these densities do not account for CT objects directly detected in X-rays. The identification of a CT spectrum in a faint X-ray source is not a straightforward task. Previous studies suggest that the fraction of CT AGNs in X-ray samples is small, of the order of a few percentages (Tozzi et al. 2006; Mainieri et al. 2007; Perola et al. 2004), so we do not try to correct our density of CT QSOs for this fraction at this stage.

\subsubsection{The Fraction of Obscured AGNs as a Function of Their Luminosity}

Table 8 also gives the volume densities of X-ray-selected AGN in the same redshift bins, computed using the parameterization of the 2-10 keV luminosity function in La Franca et al. (2005). We find that the density of infrared-selected CT QSOs at $z-1.2-2.2$ is $44 \%$ of that of all X-ray-selected AGNs in the same redshift-luminosity bin $(\sim 90 \%$ of that of both unobscured and moderately obscured QSOs). Conversely, at $z=0.7-1.2$ and $L(2-10 \mathrm{keV})=3 \times 10^{43}-10^{44}$ the density of infraredselected CT AGNs is 67\% of X-ray-selected AGNs, and $120 \%$ and $150 \%$ that of unobscured and moderately obscured AGNs, respectively. This comparison suggests that the fraction of obscured AGN to the total AGN population decreases with the luminosity not only when considering moderately obscured X-ray-selected AGNs (Ueda et al. 2003; La Franca et al. 2005), but also including infrared-selected CT AGNs.

It is also instructive to compare our estimates to the expectations of AGN synthesis models for the CXB. The expectations of the Gilli et al. (2007) model in the two luminosity bins used in our analysis are plotted in Figure 8. We see that the Gilli et al. model predicts a density of CT QSOs $(\log \mathrm{L}(2-10 \mathrm{keV})=$ 44-45), slightly higher than our estimate in the redshift bin 1.2-2.2, but consistent with it at the $90 \%$ confidence level. Conversely, the model predicts a density of $\log \mathrm{L}(2-$ $10 \mathrm{keV})=43.477-44 \mathrm{AGN}$ at $z=0.7-1.2$, a factor of $\sim 2$ lower than our estimates. However, this difference is significant at $1.5 \sigma$ level. The Gilli et al. (2007) model predicts that the fraction of obscured AGN $\left(\log \mathrm{N}_{H}>22\right.$ $\mathrm{cm}^{-2}$, including CT sources) to the total AGN population decreases with luminosity from $\sim 75 \%$ at Seyfert-like luminosities to $45 \%$ at QSO luminosities. In summary, our determinations of the CT AGN densities in two luminosity bins agree reasonably well with the Gilli et al. (2007) prediction (Figure 8). This again means that our findings support the idea that CT AGNs follow a luminosity dependence similar to Compton-thin AGNs, becoming relatively rarer at high luminosities.

\subsubsection{Comparison with Previous Studies}

We compare our estimates of the CT AGN volume density with previous determinations (see Table 9 for a summary).

Several recent papers have been focusing on the search for highly obscured AGNs in the CDFS. Using an approach similar to ours, i.e., a comparison of the ratio of the counts in stacked images in two energy bands with Monte Carlo simulations including both obscured AGN and star-forming galaxies, F08 estimated in the CDFS a density of CT AGNs with $\log$ L(2-10 $\mathrm{keV})>43$ and at $z=1.2-2.6$ similar to that of X-ray-selected AGNs. This cannot be directly compared with the densities estimated in this paper, because in the same redshift bin we can select only luminous QSOs. However, we note that the F08 estimate is similar to what we find in the C-COSMOS field in the lower redshift bin 0.7-1.2. Daddi et al. (2007), using a somewhat different approach on the same CDFS dataset, estimate that the density of CT AGNs is $\sim 2.6 \times 10^{-4} \mathrm{Mpc}^{-3}$ at $z=1.4$ 2.5 , and infer that their $2-10 \mathrm{keV}$ luminosities are in the range of $10^{43}-4 \times 10^{43}$. The Daddi et al. density is significantly higher than our estimates, and it is $\sim 6$ times higher than the expectation of the Gilli et al. (2007) model. Donley et al. (2008) recently estimated a conservative lower limit to the Spitzerselected AGNs in the CDFS, not directly detected in X-rays. They conclude that the number of AGNs with $24 \mu \mathrm{m}$ flux higher than $80 \mu \mathrm{Jy}$ is $54 \%-77 \%$ larger than for purely X-ray-selected AGNs. The fraction increases to $71 \%-94 \%$ including AGNs selected with a high radio to infrared flux ratio. The combined analysis of the COSMOS and CDFS field is clearly needed for a better coverage of the redshift-luminosity plane, and thus to measure the CT AGN luminosity function in several luminosity and redshift bins. This combined analysis will be presented in a forthcoming paper.

Alexander et al. (2008) again used Chandra deep fields, but limited their analysis to CT QSOs confirmed through infrared and optical spectroscopy, in addition to X-ray imaging. Using a small sample of four CT QSOs, they estimate a density $0.7-$ $2.5 \times 10^{-5} \mathrm{Mpc}^{-3}$ at $z=2-2.5$. This is formally higher than our estimate at a similar redshift, but still statistically consistent with it, within their rather large error bars.

Martinez-Sansigre et al. $(2005,2007)$ and Polletta et al. (2008) looked at highly obscured QSOs of extreme luminosity in the large area SWIRE, NDWFS, and FLS surveys (bolometric luminosity $\gtrsim 10^{47} \mathrm{erg} \mathrm{s}^{-1}$ ). The former authors concluded that their CT QSOs are at least as numerous as unobscured QSOs, a result similar to what we find at slightly lower luminosities in the C-COSMOS field. On the other hand, Polletta et al. (2008) conclude that $37-40 \%$ of the QSOs with $L(6 \mu \mathrm{m}) \gtrsim 4 \times 10^{45}$ erg $\mathrm{s}^{-1}$ and $z=1.3-3$ are obscured by a compact torus, while $23 \%-25 \%$ are obscured by matter distributed on larger scale. Polletta et al. (2008) do not distinguish between CT and Compton-thin absorbers; however, it is reasonable to assume that most objects obscured by a compact torus are CT, and therefore their fraction is a lower limit to the real CT fraction.

Finally, Della Ceca et al. (2008) estimated the density of CT AGNs at three luminosities, by comparing the luminosity function of optically selected narrow line AGNs in the SDSS (Simpson 2005), which must include both CT and Compton-thin AGNs, to the luminosity function of X-ray-selected Comptonthin AGNs, rescaled at $z=0$ using their best fit evolutionary 
model. The Della Ceca et al. (2008) densities are listed in Table 9. They agree quite well with the densities estimated in this paper once they are de-evolved (Della Ceca et al. 2008).

\subsection{Obscured AGN: Catching Feedback in Action}

Our findings indicate that luminous CT absorbers follow the same fundamental correlation with the luminosity found for Compton-thin absorbers. This correlation has been interpreted in the past either in terms of a luminosity dependence of the obscuring "torus" sublimation radius (e.g., Lawrence 1991), or due to the bending of the interstellar gas due to the $\mathrm{BH}$ gravitational field (the higher the $\mathrm{BH}$ mass, the larger the bending, and smaller the fraction of sight lines intercepting the gas, Lamastra et al. 2006). Recently, Menci et al. (2008) introduced a new scenario, in which the absorption properties of an AGN depend both on the orientation to the line of sight and on the time needed to sweep the central regions of galaxy disks. In this scenario, the correlation between the fraction of obscured AGNs and the luminosity is mainly due to a different timescale over which nuclear feedback is at work. If this is the case, then one may expect that X-ray selected, moderately obscured QSOs are caught at a later stage of feedback activity than highly obscured CT QSOs. Of course, this must be intended on average, because the distribution of the obscuring gas around the nucleus would not be spherically symmetric, and therefore obscuration will also depend on the orientation to the line of sight. As a consequence, some CT QSOs seen just along the plane of the obscuring material would be in a similar evolutionary stage as a moderately obscured QSO. A prediction of this evolutionary scenario is that the host galaxies of high luminosity CT QSOs should be, on average, more star forming than the host galaxies of unobscured or moderately obscured QSOs of similar luminosity. Interestingly, Stevens et al. (2005) and Page et al. (2004) find that X-ray obscured QSOs have much higher submillimeter detection rates than X-ray unobscured QSOs. Alexander et al. (2005) found that most radio identified submm galaxies host X-ray and optically obscured AGNs, but that their bolometric luminosity is dominated by star formation. Martinez-Sansigre et al. $(2005,2008)$ found little or no Lyman- $\alpha$ emission in a sample of $z>1.7$ obscured QSOs, suggesting large scale (kpc) dust distribution. Sajina et al. (2007) and Martinez-Sansigre et al. (2008) report Spitzer IRS spectra dominated by AGN continuum but showing PAHs features in emission in samples of ULIRGs and radio-selected obscured QSOs at $z \sim 2$. Finally, Lacy et al. (2007) find evidence for dust-obscured star formation in the IRS spectra of type 2 QSOs. All these findings are in general agreement with the evolutionary picture.

\subsection{Tests of the AGNs/Host Galaxy Evolutionary Scenario}

We were able to select in the C-COSMOS field both unobscured and moderately obscured AGNs using direct X-ray detection, and highly obscured CT AGNs selected in the infrared, but confirmed through a detailed X-ray stacking analysis. This makes our sample ideal to probe the above evolutionary scenario.

Star formation can be revealed by emission at radio wavelengths (Condon 1992). Radio fluxes, down to a $5 \sigma$ flux limit of $\sim 65 \mu \mathrm{Jy}$, are available for $43 \%$ of the MIPS-selected sources. The same fraction of radio fluxes is available for MIPS sources with a direct X-ray detection. Following Schinnerer et al. (2007), the $1.4 \mathrm{GHz}$ luminosity has been computed assuming a spectral energy index of 0.8 . Among the 38 sources in cells A and B and in the redshift-luminosity bin $z=1.2-2.2 \log \mathrm{L}(5.8 \mu \mathrm{m})$ $=44.79-46.18$ (see Table 7), 18 have a radio detection. Their median logarithmic radio luminosity $\left(<\log \lambda L_{\lambda}(1.4 \mathrm{GHz})\right)$ is $40.53 \mathrm{erg} \mathrm{s}^{-1}$ with interquartile range 0.18 . Putting the radio luminosity of the sources without a radio detection to the limit computed at their redshift reduces the median logarithmic radio luminosity to $40.29 \mathrm{erg} \mathrm{s}^{-1}$ with interquartile 0.18 . If the radio luminosity is due to star formation, it would imply starformation rates of the order of $300 M_{\odot} \mathrm{yr}^{-1}$ (e.g., using the correlation given by Condon 1992).

The median logarithmic ratio between the $5.8 \mu \mathrm{m}$ luminosity and the $1.4 \mathrm{GHz}$ luminosity of the 38 sources in cells A and B and in the redshift-luminosity bin $z=1.2-2.2 \log \mathrm{L}(5.8 \mu \mathrm{m})=$ 44.79-46.18 (therefore, including upper limits on the radio flux) is 4.74 with interquartile 0.12 . In the same redshift-luminosity bin there are 25 spectroscopically identified type 1 QSOs in the COSMOS bright MIPS sample, all with X-ray direct detection and eight with a radio flux. Their median radio luminosity (including limits on the radio luminosity) and the median log ratio between the infrared and radio luminosity (including limits on the radio luminosity) are 40.19 and 5.07, respectively. The probability that the luminosity ratio distribution of the 38 sources in cells A and B and the 25 type 1 QSOs are drawn from the same distribution is $0.002 \%$, using the $\mathrm{F}$ test. This would suggest a slightly stronger radio emission in infrared-selected CT QSOs than in unobscured type 1 QSOs, qualitatively in agreement with our predictions. However, we note that different components, in addition to star formation, can contribute to the observed radio flux. Furthermore, some residual extinction may still reduce the $8-24 \mu \mathrm{m}$ flux of the CT AGNs, which would then underestimate the true $5.8 \mu \mathrm{m}$ rest frame luminosity of these sources. For all these reasons, we consider the lower infrared to radio luminosity ratio of CT AGNs with respect to type 1 AGNs, certainly intriguing but not conclusive.

A cleaner test of these predictions can come from infrared spectroscopy of PAH features. Fortunately, the COSMOS infrared-selected CT AGNs are bright enough to provide relatively high signal-to-noise Spitzer IRS spectra. These spectra will be able to assess whether nuclear activity and strong star formation are present at the same time in these objects, thus validating or disproving our feedback scenario for AGN obscuration. Finally, if strong star formation is present in the host galaxies of the CT QSOs, as expected, they should stand out in forthcoming deep Herschel surveys at 70 and $110 \mu \mathrm{m}$.

\section{CONCLUSIONS}

We found that $25 \%-30 \%$ of the MIPS $24 \mu \mathrm{m}$ sources brighter than $550 \mu \mathrm{Jy}$ have a direct X-ray detection down to an X-ray flux limit of a few $\times 10^{-16} \mathrm{erg} \mathrm{cm}^{-2} \mathrm{~s}^{-1}$. About $75 \%$ of these sources are likely to be AGNs with $L(2-10) \mathrm{keV}>10^{42} \mathrm{erg} \mathrm{s}^{-1}$. We evaluated the fraction of obscured AGNs in the COSMOS MIPS sample without a direct X-ray detection by comparing the count rates and hardness ratio in stacked X-ray images with detailed Monte Carlo simulations. We found that the fraction of AGNs in this MIPS sample (both X-ray detected and recovered through their infrared/optical color and a detailed X-ray stacking analysis) is $49 \pm 10 \%$. Considering only the sources with $z>0.6$, the fraction increases to $0.67 \pm 0.06$. This is significantly higher than previous estimates obtained using a much shallower X-ray coverage and an analysis of the $24-8 \mu \mathrm{m}$ color.

We computed the volume density of the MIPS $24 \mu \mathrm{m}$-selected sources into two luminosity-redshift bins, and corrected it for 
the fraction of CT AGNs found in nine cells defined in the $F(24 \mu \mathrm{m}) / F(R)-\mathrm{R}-\mathrm{K}$ diagram to find the volume density of infrared-selected CT AGNs. Our analysis shows that deep X-ray data are the key element to obtain complete unbiased AGN samples, both through direct detection and dedicated stacking analyses. Of course, the latter can provide results on CT AGNs valid only in a statistical sense. While the search for and characterization of CT AGNs remains one of the main goals of the ongoing Chandra and XMM-Newton ultra-deep surveys of the CDFs (e.g., Comastri \& Brusa 2007), the direct $\mathrm{X}$-ray detection of large samples of CT AGNs and the accurate measure of their obscuring column densities must await for the next generation of X-ray telescopes with imaging capabilities in the 10-100 keV band, like NuSTAR, NeXT, and Simbol-X (Fiore et al. 2008b).

We found that the density of CT QSOs with $z=1.2-2.2$ and $\log \lambda L_{\lambda}(5.8 \mu \mathrm{m})=44.79-46.18$ and with $z=0.7-1.2$ and $\log \lambda L_{\lambda}(5.8 \mu \mathrm{m})=44.06-44.79$ are $\sim 44 \%$ and $\sim 67 \%$ of the density of X-ray selected unobscured and moderately obscured AGN in the same redshift and luminosity bins, respectively.

Our results imply that the correlation between the fraction of obscured AGN with the luminosity found for X-ray-selected AGNs also holds when considering infrared-selected CT AGNs. If the fraction of obscured AGNs is a measure of the timescale over which the nuclear feedback is at work, then unobscured and moderately obscured QSOs should be hosted in more passive galaxies, on average, than those hosting CT QSOs of similar luminosity. Star formation can be traced at radio wavelengths, and we find indeed that the infrared-selected CT QSOs at $z=1.2-2.2$ are more radio luminous (with respect to their $5.8 \mu \mathrm{m}$ luminosity) than unobscured type 1 QSOs of similar redshift and luminosity. Although this result is in line with previous findings, we do not consider it as conclusive. Further investigation, as for example, direct infrared spectroscopy and far infrared photometry of the candidate CT QSOs, is needed to confirm our evolutionary feedback scenario.

We acknowledge support from ASI/INAF contracts I/023/05/0 and I/024/05/0 and by PRIN/MUR grant 2006-025203. F.F. thanks Mari Polletta for useful comments. The zCOSMOS ESO Large Program Number 175.A-0839 is also acknowledged.

\section{REFERENCES}

Alexander, D. M., et al. 2005, ApJ, 632, 736

Alexander, D. M., et al. 2008, ApJ, 687, 835

Bower, et al. 2006, MNRAS, 370, 645

Boyle, B. J., Shanks, T., \& Peterson, B. A. 1988, MNRAS, 235, 935

Brand, et al. 2006, ApJ, 644, 143

Brand, et al. 2007, ApJ, 663, 204

Brandt, W. N., \& Hasinger, G. 2005, ARA\&A, 43, 1056

Brusa, M., et al. 2005, A\&A, 432, 69

Brusa, M., et al. 2007, ApJS, 172, 353

Brusa, M., et al. 2009, ApJ, in press (arXiv:0809.2513)

Capak, et al. 2007, ApJS, 172, 99

Comastri, A. 2004, in Supermassive Black Holes in the Distant Universe, vol. 308, ed. A. J. Barger (Dordrecht, The Netherlands: Kluwer), 245

Comastri, A., \& Brusa, M. 2007, Proc. Conf.: "XMM-Newton: The Next Decade," in press (arXiv:0710.0561)

Comastri, A., Gilli, R., Vignali, C., Matt, G., Fiore, F., \& Iwasawa, K. 2007, Prog. Theor. Phys. Suppl., No. 169, 274-277 (arXiv:0704.1253)

Condon, J. J. 1992, ARA\&A, 30, 575

Cowie, L., Barger, A., Bautz, M. W., Brandt, W. N., \& Garnire, G. P. 2003, ApJ, 584 , L57
Cowie, L. L., Songaila, A., Hu, E. M., \& Cohen, J. G. 1996, AJ, 112 , 839

Daddi, E., et al. 2007, ApJ, 670, 173

Della Ceca, R., et al. 2008, A\&A, 487, 119

Dey, A., et al. 2008, ApJ, 677, 943

Di Matteo, T., Springel, V., \& Hernquist, L. 2005, Nature, 433, 604

Donley, J. L., Rieke, G. H., Perez-Gonzalez, P. G., \& Barro, G. 2008, ApJ, 687, 111

Elvis, M., et al. 2009, ApJS, submitted

Fabian, A. C. 1999, MNRAS, 308, L39

Ferrarese, L., \& Merritt, D. 2000, ApJ, 539, L9

Fiore, F., et al. 2003, A\&A, 409, 79

Fiore, F., et al. 2008a, ApJ, 672, 94

Fiore, F., et al. 2008b, Proc. of the Workshop "Simbol-X: The Hard Xray Universe in Focus" Bologna 14-16 May 2007, MemSAIt, in press (arXiv:0801.0409)

Franceschini, A., Hasinger, G., Miyaji, T., \& Malguori, D. 1999, MNRAS, 310, L5

Gebhardt, K., et al. 2000, ApJ, 543, L5

Georgantopoulos, I., Georgakakis, A., Rowan-Robinson, M., \& Rovilos, E. 2008, A\&A, 484, 671

Gilli, R., Comastri, A., \& Hasinger, G. 2007, A\&A, 463, 79

Granato, G. L., De Zotti, G., Silva, L., Bressan, A., \& Danese, L. 2004, ApJ, 600,580

Granato, G. L., Silva, L., Monaco, P., Panuzzo, P., Salucci, P., De Zotti, G., \& Danese, L. 2001, MNRAS, 324, 757

Hasinger, G. 2008, A\&A, 490, 905

Hasinger, G., Miyaji, T., \& Schmidt, M. 2005, A\&A, 441, 417

Hasinger, G., et al. 2007, ApJS, 172, 29

Hopkins, A. M. 2006, in ASP Conf. Ser., ed. V. J. Alonso, H. Ferguson, \& R. Norris (arXiv:0611283)

Houck, J. R., et al. 2005, ApJ, 622, L105

Ilbert, O., et al. 2009, ApJ, 690, 1236

Lacy, M., et al. 2007, ApJ, 669, L61

La Franca, F., et al. 2005, ApJ, 635, 864

Lamastra, A., Perola, G. C., \& Matt, G. 2006, A\&A, 449, 551

Lawrence, A. 1991, MNRAS, 252, 586

Lawrence, A., \& Elvis, M. 1982, ApJ, 256, L410

Li, Y., et al. 2007, ApJ, 665, 187

Lilly, S. J., Tresse, L., Hammer, F., Crampton, D., \& Le Fevre, O. 1995, ApJ, 455,108

Lilly, S., et al. 2007, ApJS, 172, 70

Lutz, D., et al. 2004, A\&A., 418, 465

Madau, P., Ferguson, H. C., Dickinson, M. E., Giavalisco, M., Steidel, C. C., \& Fruchter, A. 1996, MNRAS, 283, 1388

Mainieri, V., et al. 2007, ApJS, 172, 368

Maiolino, R., Shemmer, O., Imanishi, M., Netzer, H., Oliva, E., Lutz, D., \& Sturm, E. 2007, A\&A, 468, 979

Maiolino, R., et al. 2001, A\&A, 365, 37

Marconi, A., Risaliti, G., Gilli, R., Hunt, L. K., Maiolino, R., \& Salvati, M. 2004, MNRAS, 351, 169

Martinez-Sansigre, A., Lacy, M., Sajina, A., \& Rawlings, S. 2008, ApJ, 674, 676

Martinez-Sansigre, A., et al. 2005, Nature, 436, 666

Martinez-Sansigre, A., et al. 2006, MNRAS, 370, 1479

Martinez-Sansigre, A., et al. 2007, MNRAS, 379, L6

Merloni, A., \& Heinz, S. 2008, MNRAS, 388, 1011

Menci, N., Fiore, F., Puccetti, S., \& Cavaliere, A. 2008, ApJ, 686, 219

Menci, N., Fontana, A., Giallongo, E., Grazian, A., \& Salimbeni, S. 2006, ApJ, 647,753

Mignoli, M., et al. 2004, A\&A, 418, 827

Page, M. J., Stevens, J. A., Ivison, R. J, \& Carrera, F. J. 2004, ApJ, 611, L85

Pope, A., et al. 2008, ApJ, 689, 127

Perola, G. C., et al. 2004, A\&A, 421, 491

Piconcelli, E., et al. 2007, A\&A, 473, 85

Polletta, M., Weedman, D., Honig, S., Lonsdale, C. J., Smith, H. E., \& Houck, J. 2008, ApJ, 675, 960

Polletta, M., et al. 2006, ApJ, 642, 673

Polletta, M., et al. 2007, ApJ, 663, 81

Pozzi, F., et al. 2007, A\&A, 468, 603

Ranalli, P., Comastri, A., \& Setti, G. 2003, A\&A, 399, 99

Renzini, A. 2006, ARA\&A, 44, 141

Salvato, M., et al. 2009, ApJ, 690, 1250

Sajina, et al. 2007, ApJ, 664, 713

Silk, J., \& Rees, M. J. 1998, A\&A, 311, L1

Sanders, D. B., et al. 2007, ApJS, 172, 86

Schmidt, M. 1968, ApJ, 151, 393 
Scoville, N., et al. 2007, ApJS, 172, 1

Schinnerer, E., et al. 2007, ApJS, 172, 46

Silva, L., Maiolino, R., \& Granato, G. L. 2004, MNRAS, 355, 973

Simpson, C. 2005, MNRAS, 360, 565

Simpson, C., Rawlings, S., \& Lacy, M. 1999, MNRAS, 306, 828

Steffen, A. T., Brandt, W. N., Alexander, D. M., Gallagher, S. C., \& Lehmer, B. D. 2007, ApJ, 667, L25

Steffen, A. T., et al. 2003, ApJL, 563, 23

Steffen, A. T., et al. 2006, AJ, 131, 2826

Stevens, J. A., et al. 2005, MNRAS, 360, 610

Tozzi, P., et al. 2006, A\&A, 451, 457
Treister, E., \& Urry, C. M. 2005, ApJ, 630, 115

Treister, E., \& Urry, C. M. 2006, ApJ, 652, L79

Treister, E., et al. 2004, ApJ, 616, 123

Trump, J., et al. 2007, ApJS, 172, 383

Trump, J., et al. 2009, ApJ, in press (arXiv:0811.3977)

Ueda, Y., et al. 2003, ApJ, 598, 886

Ueda, Y., et al. 2007, ApJ, 664, L79

Weedman, D. W., Le Floc'h, E., Higdon, S. J. U., Higdon, J. L., \& Houck, J. R. 2006a, ApJ, 638, 613

Weedman, D. W., et al. 2006b, ApJ, 651, 101

Yan, L., et al. 2007, ApJ, 658, 778 